\title{
Refractivity of Potassium Bromide for Visible Wavelengths
}

\author{
Robert J. Spindler and William S. Rodney ${ }^{2}$
}

\begin{abstract}
Values of the index of refraction of potassium bromide were determined independently on two high-precision spectrometers by the method of minimum deviation for 11 spectral lines at temperatures near $17^{\circ}, 24^{\circ}$, and $27^{\circ} \mathrm{C}$. The instruments are described, and the methods used in obtaining results valid in the sixth decimal place of index are discussed. Temperature coefficients of index were determined, and a small linear variation was found with respect to wavelength [1] ${ }^{3}$. With the values of $d n / d t$ so obtained, the experimental data were reduced to a common temperature, averaged, and a four-constant Ketteler-Helmholtz dispersion equation fitted to the data and adjusted by least squares. The refractive indices of potassium bromide for the particular prism used are intermediate between the values reported by Harting [2] and Forrest [3]. Reduced to $23^{\circ} \mathrm{C}$ for comparison, the results for sodium light are Harting, 1.55983; National Bureau of Standards, 1.55978; and Forrest, 1.55971. Another prism measured at NBS yielded 1.55983, and an obviously defective prism gave 1.55889. Small differences in dispersion were also detected.
\end{abstract}

\section{Introduction}

Potassium bromide is of interest to designers of optical instruments because of its transparancy in the infrared region of the spectrum and because of the possibility of using it in connection with other optical materials for correction of secondary spectrum. The need for precise values of refractive indices in this connection is well known. Although some data on refractive indices of potassium bromide in the visual spectral region have been published, the lack of agreement among investigators is considerable. The authors of this paper were fortunate in having the opportunity to measure the same sample independently on two high-precision spectrometers, thereby gaining a satisfactory degree of certitude regarding the validity of the results obtained, even in the sixth decimal place.

Considerable interest has been displayed by recent investigators $[4,5]$ in the thermooptical behavior of the alkali halides, primarily owing to their relatively simple crystalline structure and the information such data afford to physicists concerning interionic bond strengths. Here again, lack of precise data on temperature coefficients has handicapped physicists in theorizing upon such matters. Such considerations have led one of the authors [1] to an investigation of the dependence of index of refraction of potassium bromide upon temperature. The data thus obtained are precise enough to merit attention over the temperature range studied and are presented herein. Such data are necessary, furthermore, when reporting sixth-decimal-place values of indices of crystals whose temperature coefficients are as large as those of potassium bromide.

Investigation of the indices of different samples of the same crystalline material in the same laboratory is always of interest because of the information provided concerning control in the manufacturing

1 The work described in this paper was carried out in part under the sponsorship of the U.S. A ir Force and U.S. Army Signal Corps.

${ }^{2}$ Some of the results reported in this paper were submitted by William $\mathrm{S}$ Rodney as partial fulfillment of the requirements for the degree of Master of Science at The Catholic University of America, Washington, D. C., 1951.

3 Numbers in brackets refer to literature reference $\mathrm{a}$ a the end of this paper. process. Such information is vitally important to optical designers, as they must know the limits between which materials vary in order to make a proper choice for their design. In this regard, differences of dispersion between samples of the substance are particularly important. Measurements were performed upon three different samples of potassium bromide grown by the Harshaw Chemical Corporation. Two of the samples were finished into $60^{\circ}$ prisms, and the third was finished into a $30^{\circ}$ prism by the Perkin-Elmer Corporation. The two $60^{\circ}$ prisms were made available to the authors by Earle K. Plyler of the Radiometry Section of the National Bureau of Standards. The prism measured upon two spectrometers at three temperatures was one of these. The $30^{\circ}$ prism was made available to the authors by Stanley S. Ballard and the Polaroid Corporation.

\section{Description of Apparatus}

\subsection{Watts Spectrometer}

One of the spectrometers (fig. 1) was made by E. R. Watts \& Son, Ltd., of London, and is based on the original National Physical Laboratory design described in detail in the literature [6]. The collimator and telescope objectives have focal lengths of $60 \mathrm{~cm}$ and $40 \mathrm{~cm}$, and clear apertures of $9 \mathrm{~cm}$ and 4 $\mathrm{cm}$, respectively. An auxiliary telescope, identical in every respect to the rotating telescope, and mounted on a pedestal that may be clamped to the circular base, is furnished with the instrument. It may be used for a variety of purposes, including adjustment of collimation, measurement of prism angles by the substitution method, and measurement of the error in the $90^{\circ}$ deviation of optical squares.

The collimator slit of the instrument is provided with a device for opening and closing the slit jaws symmetrically. A graduated drum is attached to the slit-width adjusting screw in order that slit widths can be recorded and repeated when necessary. Two parallel vertical lines bisected by a horizontal line are etched upon a glass plate that is mounted im- 


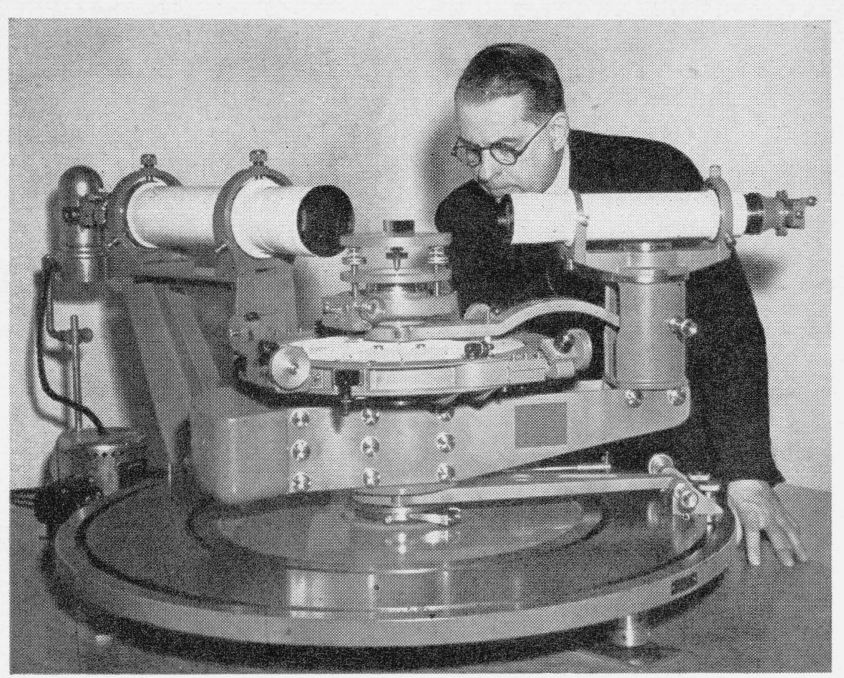

FIguRE 1. Watts spectrometer.

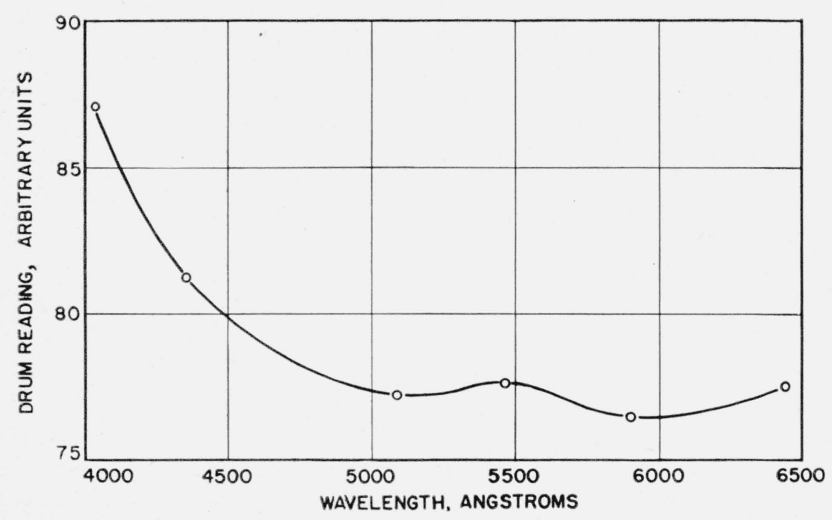

Figure 2. Tube lengths for perfect collimation of telescope of Watts spectrometer.

mediately behind the slit, that is, upon the source side. In adjusting collimation and making pointings, focus is made upon these lines in order that wide slits may be used to obtain sufficient intensity and to reduce chromatic parallax, as recommended by Guild [7].

Both telescopes are provided with rotatable eyepiece micrometers, permitting rapid and accurate determination of small angles and pyramidal errors of prisms. Several eyepieces of various powers are provided, and one of the Gauss type is used for leveling and autocollimation.

A removable table containing two slides that travel in superimposed ways at right angles to each other was constructed for the prism table of the instrument. The slides are actuated by slow-motion screws and greatly facilitate centering the prism with respect to collimator and telescope objectives.

Both telescopes and the collimator are provided with a rack-and-pinion device for focusing purposes. A graduated circular drum is mounted upon the pinion gear and positions of the rack may be noted by means of the graduations. By means of a monochromator manufactured by the Carl Zeiss Co. of

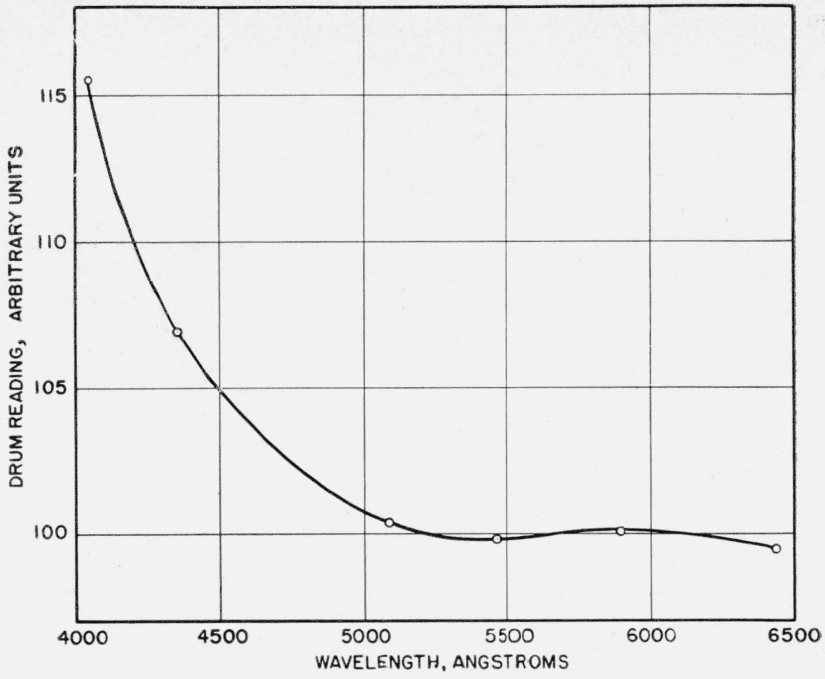

Figure 3. Tube lengths for perfect collimation of auxiliary telescope of Watts spectrometer.

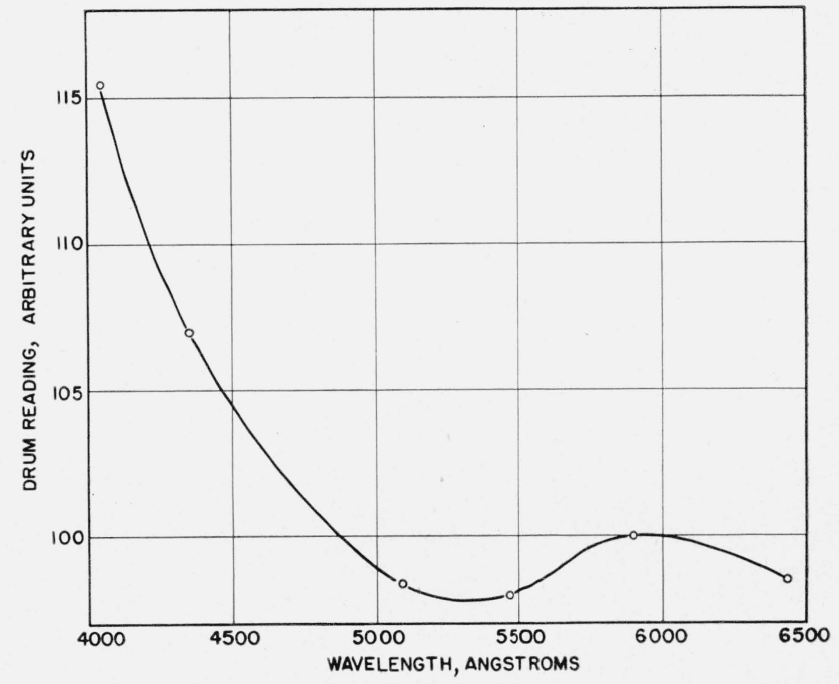

Figure 4. Tube lengths for perfect collimation of collimator of Watts spectrometer.

Jena, fitted with a flint-glass prism as dispersing agent, several spectral lines of a cadmium-mercury FH-4 lamp were separated and used as sources to illuminate the collimator slit. All three tube lengths were adjusted for truly parallel monochromatic light of each particular wavelength by the usual method of focusing upon each other in pairs, as described by Glazebrook [8]. The drum readings were recorded, and the results are shown in figures 2,3 , and 4 . With the aid of these data the collimator and telescope may be adjusted for truly parallel monochromatic light. If the prism has appreciable power, owing, for example, to eurved surfaces, this will be evidenced by the modification of telescope tube length necessary for sharp focus of the collimator-slit image. 


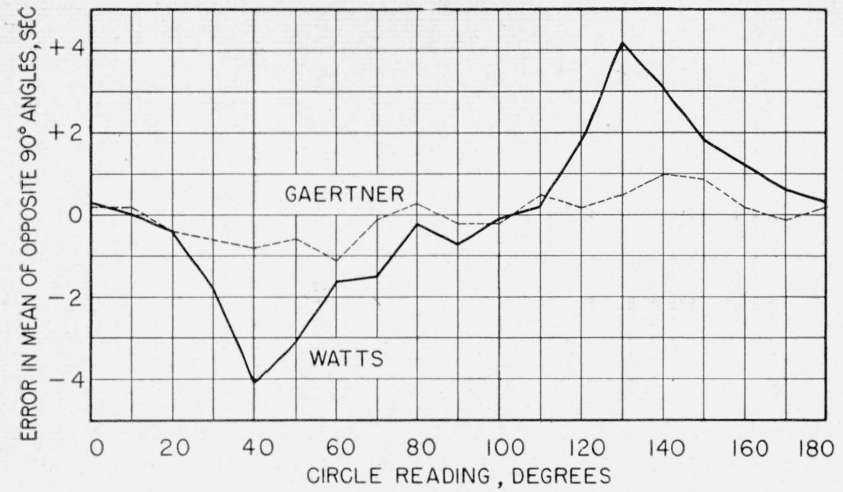

FIGURE 5. Error in the mean of opposite $90^{\circ}$ angles as a function of circle orientation.

The microscopes are mounted on a ring that may be rotated independently around the circle. This greatly facilitates the reading of the microscopes, for their positions may be so adjusted as to eliminate the troublesome cases where the microscopes are inaccessible for certain telescope orientations. The micrometer screws make 1 revolution per minute of arc, and the drums are graduated to read halfseconds directly. The elimination of parallax was effected by proper focusing, and the tube length of each microscope was adjusted to insure proper magnification so that 5 revolutions of the drum covered the average of a few 5-min intervals ruled upon the circle.

The divided circle is ruled on the periphery of a 15-in. cylindrical drum and is graduated to 5 -min intervals. A complete calibration of the circle graduations has not been made, but a test showing the error in the mean of readings for opposite angles caused by positional errors of the degree graduations has been made at $10^{\circ}$ intervals completely around the circle. For convenience, the size of the opposite angles was chosen to be $90^{\circ}$. The results of this test are shown in figure 5 . The maximum value of 4.2 sec is uncomfortably large, but as is more or less well known [9], the effect of positional error of the circle markings upon measurements can be satisfactorily minimized in the average, provided a systematic program of observations using proper orientations of the circle is followed.

Errors of subintervals of the degree intervals were determined by measurements on four $1^{\circ}$ intervals near $0^{\circ}, 90^{\circ}, 180^{\circ}$, and $270^{\circ}$, respectively. These measurements show that there is a periodic error that repeats six times in each degree interval and has an amplitude of approximately 1 sec. The periodic nature of these errors is such that their effect will be largely minimized in the average of two microscopes by making their angular displacement $179^{\circ} 55^{\prime}$ instead of exactly $180^{\circ}$. Thus in figure 6 the errors of the degree subintervals as seen under microscope A are shown by the solid line. If corresponding intervals of the circle pass under microscopes $A$ and $B$ simultaneously, the degree subinterval errors seen under microscope B would be identical with those seen under $\mathrm{A}$, and no reduction would be possible by

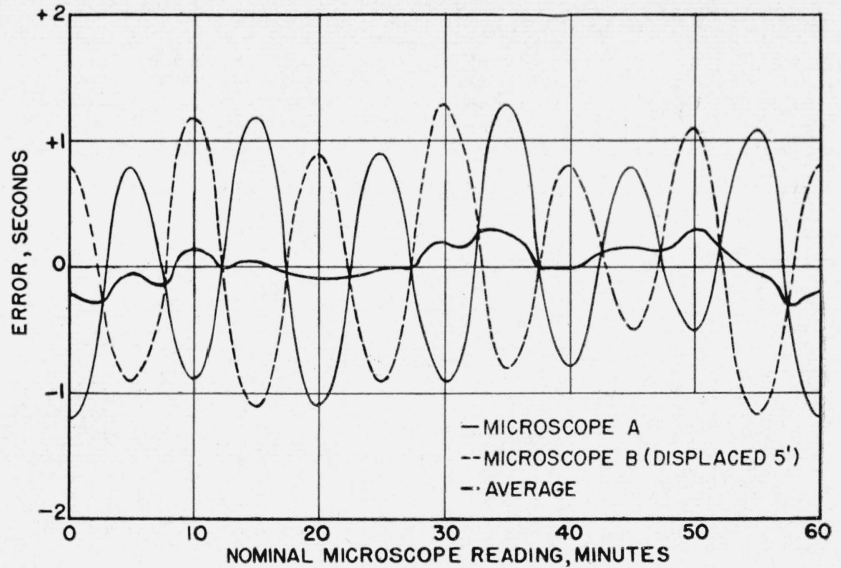

Figure 6. Error of degree subintervals as a function of microscope reading on initial graduation of each 5-minute subinterval (Watts spectrometer).

averaging $\mathrm{A}$ and $\mathrm{B}$. If, however, the phase relationship of the error is altered by displacing microscope $\mathrm{B}$ one-half period $(5 \mathrm{~min})$, then the errors of the degree subintervals, as they will appear under B, are shown by the dotted line in figure 6 . The residual error after averaging both microscopes will be reduced to the amount shown by the heavy line.

The report of an examination by the National Physical Laboratory of the circle of a similar spectrometer manufactured by the same firm is of interest because one might suspect the general characteristics of the two circles to be similar, assuming that both were ruled upon the same engine. This report mentions a systematic error that "recurs every ten minutes of arc and which results in a mean disparity of $1.05( \pm 0.1)$ seconds in the alternate spaces between adjacent graduations." This is adequately descriptive of the error of the degree subintervals found for the instrument in the NBS laboratory. The report further states that the main systematic error can be represented "by a periodic error in the graduations of which only half a complete period is comprised within the full circle of the scale. The beginning and end of this half-period coincides closely with the zero graduation." Designating by $\theta$ an angle measured from the scale zero, the report further states "The error is zero for $\theta=0^{\circ}, 180^{\circ}$, or $360^{\circ}$ and has a maximum value of +1.95 seconds for $\theta=90^{\circ}$ or $270^{\circ}$. The error is positive for all values of $\theta$, the errors of the second half of the scale $\left(180^{\circ}\right.$ to $\left.360^{\circ}\right)$ being a repetition of those of the first half without change of sign." "The rms value of the random component of the error of position of a graduation is $0.60 \pm 0.07$ seconds."

The test performed here on the circle of the $\mathrm{Bu}$ reau's instrument does not yield directly comparable information, but it is of interest to estimate the results that would probably be obtained by testing the circle referred to in the NPL report in the same manner in which the circle mounted in the instrument in the Bureau's laboratory was tested. The periodicity of the systematic error is the same for both circles. In making a $90^{\circ}$ opposite-angle test on the circle 


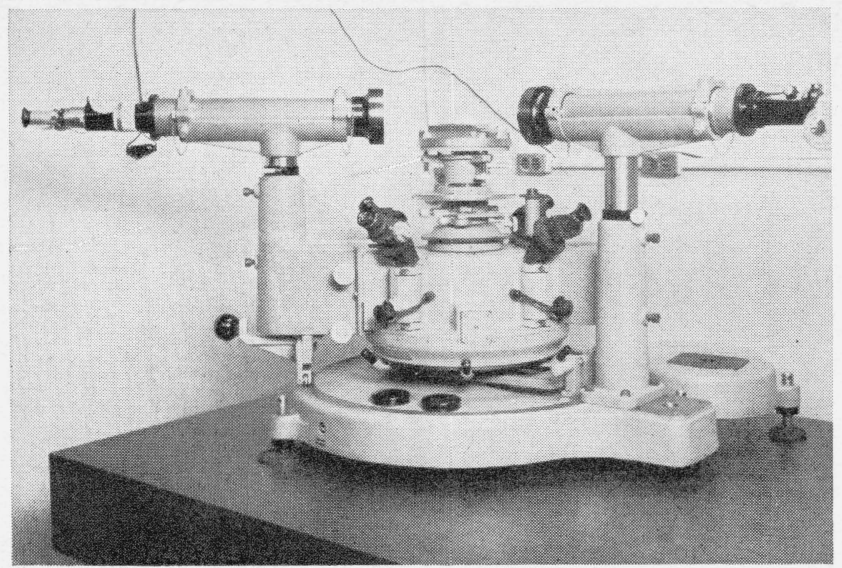

Figure 7. Gaertner spectrometer.

described in the NPL report, the error would be 0 for one $90^{\circ}$ angle as read between $315^{\circ}$ and $45^{\circ}$ (the other $90^{\circ}$ angle read from $165^{\circ}$ to $225^{\circ}$ ) and +1.95 sec for one $90^{\circ}$ angle as read between $0^{\circ}$ and $90^{\circ}$ (the other $90^{\circ}$ angle read between $180^{\circ}$ and $270^{\circ}$ ). The superposed random component of $\mathrm{rms}= \pm 0.60 \mathrm{sec}$ for line position contributes an rms random component of 0.60 sec for the mean of opposite-angle readings. If it chances that the random component should be as large as twice the rms value for lines used when the first $90^{\circ}$ angle is read in the vicinity of $0^{\circ}$ to $90^{\circ}$, then an error of +3.2 sec would be found, as compared with +4 sec, the extreme found on the instrument tested here.

Thus it would appear that the over-all quality of the circle reported upon by the NPL may be somewhat higher than the one tested at the NBS. Nevertheless, the characteristics of these circles with respect to magnitude and periodicity of errors are very similar.

\subsection{Gaertner Spectrometer}

The other spectrometer (fig. 7) was made by the Gaertner Scientific Corp., Chicago, Ill. It is designated as type $\mathrm{L}-124$ and is of recent design. The telescope and collimator each have focal lengths of $50 \mathrm{~cm}$ and a clear aperture of $5 \mathrm{~cm}$. Several eyepieces of various powers are provided, including a Gaussian used for leveling and autocollimation. Slow-motion screws are provided for making small angular displacements of the telescope and the divided circle. The collimator slit is provided with an auxiliary prism to facilitate comparison of spectra. A vertical spider thread is mounted in the center of the collimator slit, so that wide slits could be used and pointings made upon the spider thread in order to lessen chromatic parallax [7]. A removable table, similar to the one described in section 2.1, is mounted upon the prism table of the instrument.

The circle, which is of stainless steel, has a diameter of $300 \mathrm{~mm}$. A zone about $1 \mathrm{in}$. wide near the periphery of the circle was ground and polished optically flat by the Bureau's Optical Shop. Lines separated by $5 \mathrm{~min}$ of arc were ruled upon the polished zone by Benjamin L. Page in the Length Sec-

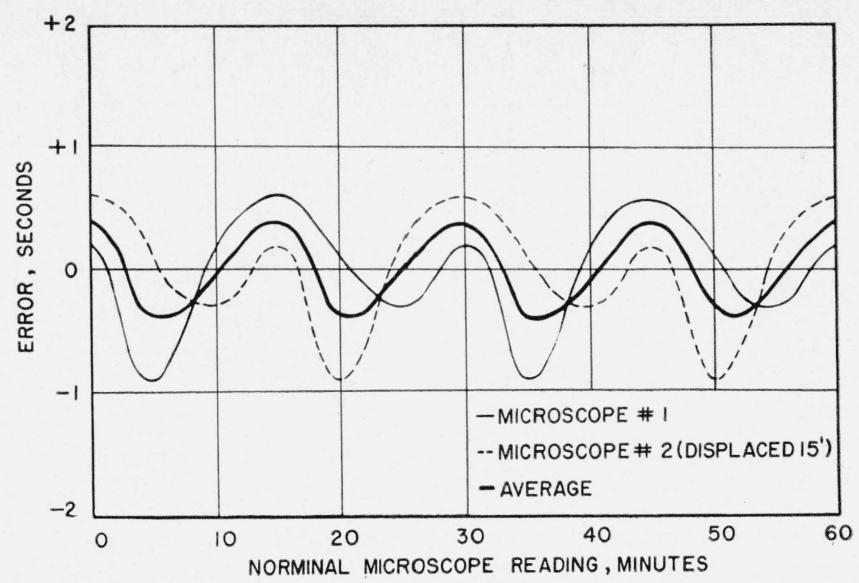

FIguRE 8. Error of degree subintervals as a function of microscope reading on initial graduation of each 5-minute subininterval (Gaertner spectrometer).

tion of the National Bureau of Standards. The errors of position of the degree graduations have not been explicitly investigated, but a test of the error of the mean of opposite $90^{\circ}$ angles, similar to that described in section 2.1, was made. The results of this test are plotted in figure 5. The maximum value of $1.1 \mathrm{sec}$ indicates that the effect of positional errors of the degree markings are much less serious, approximately one-fourth of those on the Watts instrument. The same systematic program of observations mentioned in section 2.1 was used in making all determinations on this instrument.

The results of an investigation of errors in the degree subintervals are shown in figure 8 . The error of the intervals, although not so completely periodic as was the case with the circle of the Watts instrument, appears to repeat itself twice in each degree interval and has a maximum amplitude of $0.9 \mathrm{sec}$ of arc. By making the angular separation of two micrometer microscopes $179^{\circ} 45^{\prime}$ instead of exactly $180^{\circ}$, these errors will be largely minimized in their average, as discussed in section 2.1. Thus in figure 8 the errors as seen under microscope 1 are shown by the solid line. If microscope 2 is displaced one-half period $(15 \mathrm{~min})$ so that the errors seen under it are of the amounts indicated by the dotted line, the amplitude of the residual error in the average of the two will be reduced to a uniform value of $0.4 \mathrm{sec}$, as shown by the heavy line.

\section{Experimental Program and Procedure}

Index determinations were made at temperatures near $17^{\circ}, 22^{\circ}$, and $27^{\circ} \mathrm{C}$ for 11 spectral lines. The sample was kept in a dessicator while not actually being measured. The relative humidity of the room in which the measurements were made was regulated so as not to exceed 45 percent. No deterioration of the surfaces of the sample became noticeable during the course of the experiments. Care was taken to allow the sample sufficient time to reach temperature equilibrium before making deviation measurements. 
Fifteen or more hours were allowed for reaching temperature equilibrium whenever the temperature of the surroundings was changed.

All refracting-angle determinations were made by autocollimation, with the Gauss eyepieces supplied with the instruments. Measurements at each of the three temperatures failed to demonstrate any temperature dependence of the refracting angle. In order to minimize torsional errors of the cone, the procedure recommended by Tilton [9] was followed, one-half the measurements being made on the angle itself and the others on its explement. ${ }^{4}$ The difference between the average of these two sets of data was observed to be negligible on both spectrometers at $22^{\circ} \mathrm{C}$ and $27^{\circ} \mathrm{C}$ but amounted to about $1.5 \mathrm{sec}$ on the Gaertner instrument at the lower temperature. The refracting angle was of the order of magnitude of $60^{\circ}$. Symmetrical use of the circle was achieved by using three different orientations displaced $60^{\circ}$ from each other for each set of angle measurements.

Measurements of double deviation were made for each spectral line after the prismatic sample had been centered with respect to the telescope and collimator objectives. Because the double deviation was always of the order of $90^{\circ}$, two determinations with the circle orientations $90^{\circ}$ apart suffice to cancel approximately the effects of positional errors of the circle rulings. At least two, and sometimes four, individual determinations of double deviation were made on each instrument for each spectral line measured.

The temperature and the humidity of the laboratory in which the measurements were made were automatically controlled. The prism temperature was assumed to be the same as that recorded by a mercury thermometer suspended directly over the prism, with the mercury bulb almost in contact. The temperature was recorded at equal time intervals during the course of the observations by an observer viewing the thermometer through a telescope. The thermometer could be read directly to the nearest tenth of a degree, and the hundredths estimated. The thermometer was calibrated by the Bureau's Thermometry Section, and the correction applied over the range used was negligible. Care was taken to shield the thermometer from sources of radiation. The light source was located in a fiberboard container, and the room was more or less darkened while the observations were being made. Fluctuations in temperature were noted because of the unavoidable proximity of the observer operating the spectrometer. The average deviation of the recorded temperatures was $0.03^{\circ} \mathrm{C}$. The room temperature was adjusted to the desired value, and after sufficient time had elapsed for equilibrium conditions to prevail, the deviation measurements were made.

All indices reported in this paper were corrected to refer to dry air at $760-\mathrm{mm}$ pressure $(\mathrm{Hg})$, and to the air temperature listed, by use of tables and procedures described by Tilton [10]. Temperature coefficients were computed for each of the 11 wave-

\footnotetext{
4 "Explement" is defined as the angle that must be added to a given angle to produce 360 degrees.
}

lengths and plotted. A straight line was passed through these data, and a least-squares adjustment of the constants was made. With the values of $d n / d t$ computed from this equation, (2), all observed data were adjusted to $22^{\circ} \mathrm{C}$. A four-constant KettelerHelmholtz dispersion equation was fitted to the weighted mean of the observed data and adjusted by the method of least squares.

\section{Results}

Values of refractive index at $22^{\circ} \mathrm{C}$ were computed for wavelengths ranging from 4000 to $7100 \mathrm{~A}$ from the dispersion equation

$$
n^{2}=2.3618102-0.00058072 \lambda^{2}+\frac{0.02305269}{\lambda^{2}-0.0245381}
$$

and for temperatures ranging from $16^{\circ}$ to $28^{\circ} \mathrm{C}$ from the temperature coefficient equation

$$
\frac{d n}{d t}=-(31.742+13.21 \lambda) \times 10^{-6}
$$

where $\lambda$ is in microns. These values are listed in table 1. The tabular intervals are so chosen that sixth-decimal-place indices may be obtained by linear interpolation. Table 2 lists light sources, spectral lines, wavelengths, observed and computed indices, and their differences. Table 3 lists values of $d n / d t$ observed and computed and their differences.

Figure 9 shows a comparison of the values reported by other investigators $[2,3,11,12,13]$. The ordinates represent differences between the values of other observers and values reported in table 1 of this paper. Christiansen's values, like Sprockhoff's are only for the C, D, and F lines. Gyulai's values were determined on an instrument using a physical detector. He was chiefly interested in the ultraviolet indices, and the visible data represent one extreme of his observations. The most recently reported values are those of Forrest [3] and Harting [2]. The average difference at $20^{\circ} \mathrm{C}$ between Forrest's values and the authors' is approximately $-7 \times 10^{-5}$ and is constant within $\pm 5 \times 10^{-6}$; and between Harting's values and the authors' the difference is $+4 \times 10^{-5}$. The constancy in the sixth decimal place between Forrest's values and those of the authors' indicate that the internal precision of both sets of data is very high. The constancy with respect to Harting's data is of the order of one unit in the fifth decimal place, indicating a somewhat lesser degree of precision in his work. The average difference between the values reported by Forrest and by Harting is of the order of one unit in the fourth decimal place. Both these sets of data were determined in comparatively recent times on high-precision spectrometers by using modern techniques, and the differences between the two sets of data are probably caused by varying amounts of impurities in the samples themselves. 
TABLE 1. Refractive indices of potassium bromide

[Computed from equations 1 and 2]

\begin{tabular}{|c|c|c|c|c|c|c|c|c|c|c|c|c|c|}
\hline \multirow{2}{*}{ Wavelength } & \multicolumn{13}{|c|}{ Refractive index at - } \\
\hline & $16^{\circ} \mathrm{C}$ & $17^{\circ} \mathrm{C}$ & $18^{\circ} \mathrm{C}$ & $19^{\circ} \mathrm{C}$ & $20^{\circ} \mathrm{C}$ & $21^{\circ} \mathrm{C}$ & $22^{\circ} \mathrm{C}$ & $23^{\circ} \mathrm{C}$ & $24^{\circ} \mathrm{C}$ & $25^{\circ} \mathrm{C}$ & $26^{\circ} \mathrm{C}$ & $27^{\circ} \mathrm{C}$ & $28^{\circ} \mathrm{C}$ \\
\hline $\begin{array}{l}\quad A \\
4000 \\
4010 \\
4020 \\
40030 \\
4040\end{array}$ & $\begin{array}{l}1.591415 \\
1.591101 \\
1.590789 \\
1.590480 \\
1.590174\end{array}$ & $\begin{array}{l}\text { 1. } 591378 \\
\text { 1. } 591064 \\
\text { 1. } 590752 \\
\text { 1. } 590443 \\
1.590137\end{array}$ & $\begin{array}{l}1.591341 \\
1.591027 \\
1.590715 \\
1.590406 \\
1.590100\end{array}$ & $\begin{array}{l}1.591304 \\
1.590990 \\
1.590678 \\
1.590369 \\
1.590063\end{array}$ & $\begin{array}{l}\text { 1. } 591267 \\
1.590953 \\
1.590641 \\
1.590332 \\
1.590026\end{array}$ & $\begin{array}{l}1.591230 \\
1.590916 \\
1.590604 \\
1.590295 \\
1.589989\end{array}$ & $\begin{array}{l}\text { 1. } 591193 \\
\text { 1. } 590879 \\
\text { 1. } 590567 \\
\text { 1. } 590258 \\
\text { 1. } 589952\end{array}$ & $\begin{array}{l}1.591156 \\
1.590842 \\
1.590530 \\
1.590221 \\
1.589915\end{array}$ & $\begin{array}{l}1.591119 \\
1.590805 \\
1.590493 \\
1.590184 \\
1.589878\end{array}$ & $\begin{array}{l}1.591082 \\
1.590768 \\
1.590456 \\
1.590147 \\
1.589841\end{array}$ & $\begin{array}{l}1.591045 \\
1.590731 \\
1.590419 \\
1.590110 \\
1.589804\end{array}$ & $\begin{array}{l}1.591008 \\
1.590694 \\
1.590382 \\
1.590073 \\
1.589767\end{array}$ & $\begin{array}{l}\text { 1. } 590971 \\
\text { 1. } 590657 \\
\text { 1. } 590345 \\
1.590036 \\
1.589730\end{array}$ \\
\hline $\begin{array}{l}4050 \ldots \\
4060 \ldots \\
4070 \ldots \\
4080 \\
4090\end{array}$ & $\begin{array}{l}\text { 1. } 589872 \\
\text { 1. } 589571 \\
\text { 1. } 589273 \\
\text { 1. } 588978 \\
1.588685\end{array}$ & $\begin{array}{l}\text { 1. } 589834 \\
\text { 1. } 589534 \\
\text { 1. } 589236 \\
\text { 1. } 588941 \\
\text { 1. } 588648\end{array}$ & $\begin{array}{l}\text { 1. } 589797 \\
\text { 1. } 589496 \\
\text { 1. } 589198 \\
\text { 1. } 588904 \\
1.588611\end{array}$ & $\begin{array}{l}\text { 1. } 589760 \\
1.589459 \\
1.589161 \\
1.588866 \\
1.588573\end{array}$ & $\begin{array}{l}1.589723 \\
1.589422 \\
1.589124 \\
1.588829 \\
1.588536\end{array}$ & $\begin{array}{l}\text { 1. } 589686 \\
\text { 1. } 589385 \\
\text { 1. } 589087 \\
\text { 1. } 588792 \\
\text { 1. } 588499\end{array}$ & $\begin{array}{l}\text { 1. } 589649 \\
1.589348 \\
1.589050 \\
1.588755 \\
1.588462\end{array}$ & $\begin{array}{l}\text { 1. } 589612 \\
1.589311 \\
1.589013 \\
1.588718 \\
1.588425\end{array}$ & $\begin{array}{l}\text { 1. } 589575 \\
\text { 1. } 589274 \\
\text { 1. } 588976 \\
\text { 1. } 588681 \\
\text { 1. } 588388\end{array}$ & $\begin{array}{l}\text { 1. } 589538 \\
\text { 1. } 589237 \\
1.588939 \\
\text { 1. } 588644 \\
1.588351\end{array}$ & $\begin{array}{l}1.589501 \\
1.589200 \\
1.588902 \\
1.588606 \\
1.588313\end{array}$ & $\begin{array}{l}\text { 1. } 589464 \\
\text { 1. } 589162 \\
\text { 1. } 588864 \\
\text { 1. } 588569 \\
1.588276\end{array}$ & $\begin{array}{l}\text { 1. } 589426 \\
\text { 1. } 589125 \\
\text { 1. } 588827 \\
\text { 1. } 588532 \\
1.588239\end{array}$ \\
\hline $\begin{array}{l}4100_{-} \\
4110_{-} \\
4120 \\
4130 \\
4140\end{array}$ & $\begin{array}{l}\text { 1. } 588395 \\
\text { 1. } 588107 \\
\text { 1. } 587822 \\
\text { 1. } 587539 \\
1.587259\end{array}$ & $\begin{array}{l}\text { 1. } 588358 \\
\text { 1. } 588070 \\
1.587785 \\
\text { 1. } 587502 \\
1.587222\end{array}$ & $\begin{array}{l}\text { 1. } 588321 \\
\text { 1. } 588033 \\
\text { 1. } 587748 \\
1.587465 \\
1.587185\end{array}$ & $\begin{array}{l}1.588283 \\
1.587996 \\
1.587711 \\
1.587428 \\
1.587148\end{array}$ & $\begin{array}{l}1.588246 \\
1.587958 \\
1.587673 \\
1.587390 \\
1.587110\end{array}$ & $\begin{array}{l}\text { 1. } 588209 \\
1.587921 \\
1.587636 \\
1.587353 \\
1.587073\end{array}$ & $\begin{array}{l}\text { 1. } 588172 \\
1.587884 \\
1.587599 \\
1.587316 \\
1.587036\end{array}$ & $\begin{array}{l}\text { 1. } 588135 \\
\text { 1. } 587847 \\
1.587562 \\
1.587279 \\
1.586999\end{array}$ & $\begin{array}{l}\text { 1. } 588098 \\
\text { 1. } 587810 \\
\text { 1. } 587525 \\
\text { 1. } 587242 \\
\text { 1. } 586962\end{array}$ & $\begin{array}{l}\text { 1. } 588061 \\
\text { 1. } 587772 \\
\text { 1. } 587487 \\
\text { 1. } 587204 \\
\text { 1. } 586924\end{array}$ & $\begin{array}{l}1.588023 \\
1.587735 \\
1.587450 \\
1.587167 \\
1.586887\end{array}$ & $\begin{array}{l}1.587986 \\
1.587698 \\
1.587413 \\
1.587130 \\
1.586850\end{array}$ & $\begin{array}{l}\text { 1. } 587949 \\
\text { 1. } 587661 \\
1.587376 \\
\text { 1. } 587093 \\
1.586813\end{array}$ \\
\hline $\begin{array}{l}4150 \ldots \\
4160- \\
4170- \\
4180- \\
4190_{-}\end{array}$ & $\begin{array}{l}\text { 1. } 586981 \\
1.586706 \\
1.586434 \\
\text { 1. } 586163 \\
1.585895\end{array}$ & $\begin{array}{l}\text { 1. } 586944 \\
\text { 1. } 586669 \\
\text { 1. } 586396 \\
\text { 1. } 586125 \\
1.585857\end{array}$ & $\begin{array}{l}\text { 1. } 586907 \\
\text { 1. } 586632 \\
\text { 1. } 586359 \\
\text { 1. } 586088 \\
1.585820\end{array}$ & $\begin{array}{l}1.586870 \\
1.586595 \\
1.586322 \\
1.586051 \\
1.585783\end{array}$ & $\begin{array}{l}\text { 1. } 586832 \\
1.586557 \\
1.586285 \\
1.586014 \\
1.585746\end{array}$ & $\begin{array}{l}\text { 1. } 586795 \\
1.586520 \\
1.586247 \\
1.585976 \\
1.585708\end{array}$ & $\begin{array}{l}\text { 1. } 586758 \\
1.586483 \\
1.586210 \\
1.585939 \\
1.585671\end{array}$ & $\begin{array}{l}\text { 1. } 586721 \\
1.586446 \\
1.586173 \\
\text { 1. } 585902 \\
1.585634\end{array}$ & $\begin{array}{l}\text { 1. } 586684 \\
\text { 1. } 586409 \\
\text { 1. } 586136 \\
\text { 1. } 585864 \\
\text { 1. } 585596\end{array}$ & $\begin{array}{l}\text { 1. } 586646 \\
\text { 1. } 586371 \\
1.586098 \\
1.585827 \\
1.585559\end{array}$ & $\begin{array}{l}1.586609 \\
1.586334 \\
1.586061 \\
1.585790 \\
1.585522\end{array}$ & $\begin{array}{l}\text { 1. } 586572 \\
\text { 1. } 586297 \\
\text { 1. } 586024 \\
\text { 1. } 585753 \\
1.585485\end{array}$ & $\begin{array}{l}\text { 1. } 586535 \\
\text { 1. } 586260 \\
1.585987 \\
1.585715 \\
1.585447\end{array}$ \\
\hline $\begin{array}{l}4200 \\
4210^{-} \\
4220 \\
4230 \\
4240\end{array}$ & $\begin{array}{l}1.585629 \\
1.585365 \\
1.585103 \\
1.584844 \\
1.584587\end{array}$ & $\begin{array}{l}1.585591 \\
1.585328 \\
1.585066 \\
1.584807 \\
1.584550\end{array}$ & $\begin{array}{l}1.585554 \\
1.585290 \\
1.585028 \\
1.584769 \\
1.584512\end{array}$ & $\begin{array}{l}1.585517 \\
1.585253 \\
1.584991 \\
1.584732 \\
1.584475\end{array}$ & $\begin{array}{l}1.585480 \\
1.585216 \\
1.584954 \\
1.584695 \\
1.584438\end{array}$ & $\begin{array}{l}\text { 1. } 585442 \\
\text { 1. } 585178 \\
\text { 1. } 584916 \\
1.584657 \\
1.584400\end{array}$ & $\begin{array}{l}1.585405 \\
1.585141 \\
1.584879 \\
1.584620 \\
1.584363\end{array}$ & $\begin{array}{l}\text { 1. } 585368 \\
1.585104 \\
1.584842 \\
1.584583 \\
1.584326\end{array}$ & $\begin{array}{l}1.585330 \\
1.585066 \\
1.584804 \\
1.584545 \\
1.584288\end{array}$ & $\begin{array}{l}\text { 1. } 585293 \\
\text { 1. } 585029 \\
\text { 1. } 584767 \\
1.584508 \\
1.584251\end{array}$ & $\begin{array}{l}1.585256 \\
1.584992 \\
1.584730 \\
1.584471 \\
1.584214\end{array}$ & $\begin{array}{l}\text { 1. } 585219 \\
\text { 1. } 584954 \\
\text { 1. } 584692 \\
\text { 1. } 584433 \\
\text { 1. } 584176\end{array}$ & $\begin{array}{l}\text { 1. } 585181 \\
1.584917 \\
1.584655 \\
1.584396 \\
1.584139\end{array}$ \\
\hline $\begin{array}{l}4250 \text { - } \\
4260 \text { - } \\
4270- \\
4280_{-} \\
4290_{-}\end{array}$ & $\begin{array}{l}1.584332 \\
1.584078 \\
1.583828 \\
1.583579 \\
1.583332\end{array}$ & $\begin{array}{l}\text { 1. } 584295 \\
\text { 1. } 584041 \\
\text { 1. } 583791 \\
\text { 1. } 583542 \\
1.583295\end{array}$ & $\begin{array}{l}1.584257 \\
1.584003 \\
1.583754 \\
1.583505 \\
1.583258\end{array}$ & $\begin{array}{l}\text { 1. } 584220 \\
1.583966 \\
1.583716 \\
1.583467 \\
1.583220\end{array}$ & $\begin{array}{l}1.584183 \\
1.583929 \\
1.583679 \\
1.583430 \\
1.583183\end{array}$ & $\begin{array}{l}1.584145 \\
1.583891 \\
1.583641 \\
1.583392 \\
1.583145\end{array}$ & $\begin{array}{l}\text { 1. } 584108 \\
\text { 1. } 583854 \\
\text { 1. } 583604 \\
\text { 1. } 583355 \\
\text { 1. } 583108\end{array}$ & $\begin{array}{l}\text { 1. } 584071 \\
\text { 1. } 583817 \\
\text { 1. } 583567 \\
1.583318 \\
1.583071\end{array}$ & $\begin{array}{l}\text { 1. } 584033 \\
\text { 1. } 583779 \\
\text { 1. } 583529 \\
\text { 1. } 583280 \\
\text { 1. } 583033\end{array}$ & $\begin{array}{l}\text { 1. } 583996 \\
1.583742 \\
1.583492 \\
1.583243 \\
1.582996\end{array}$ & $\begin{array}{l}1.583959 \\
1.583705 \\
1.583454 \\
1.583205 \\
1.582958\end{array}$ & $\begin{array}{l}1.583921 \\
1.583667 \\
1.583417 \\
1.583168 \\
1.582921\end{array}$ & $\begin{array}{l}\text { 1. } 583884 \\
1.583630 \\
1.583380 \\
1.583131 \\
1.582884\end{array}$ \\
\hline $\begin{array}{l}4300_{\ldots} \\
4310_{\ldots} \\
4320_{-} \\
4330 \\
4340_{-}\end{array}$ & $\begin{array}{l}\text { 1. } 583089 \\
1.582846 \\
1.582605 \\
1.582367 \\
1.582130\end{array}$ & $\begin{array}{l}\text { 1. } 583051 \\
1.582808 \\
1.582567 \\
1.582329 \\
1.582092\end{array}$ & $\begin{array}{l}1.583014 \\
1.582771 \\
1.582530 \\
1.582292 \\
1.582055\end{array}$ & $\begin{array}{l}1.582976 \\
1.582733 \\
1.582492 \\
1.582254 \\
1.582017\end{array}$ & $\begin{array}{l}1.582939 \\
1.582696 \\
1.582455 \\
1.582217 \\
1.581980\end{array}$ & $\begin{array}{l}\text { 1. } 582901 \\
1.582658 \\
1.582417 \\
1.582179 \\
1.581942\end{array}$ & $\begin{array}{l}\text { 1. } 582864 \\
\text { 1. } 582621 \\
\text { 1. } 582380 \\
\text { 1. } 582142 \\
1.581905\end{array}$ & $\begin{array}{l}1.582827 \\
1.582584 \\
1.582343 \\
1.582105 \\
1.581868\end{array}$ & $\begin{array}{l}\text { 1. } 582789 \\
\text { 1. } 582546 \\
\text { 1. } 582305 \\
\text { 1. } 582067 \\
1.581830\end{array}$ & $\begin{array}{l}1.582752 \\
1.582509 \\
1.582268 \\
1.582030 \\
1.581793\end{array}$ & $\begin{array}{l}1.582714 \\
1.582471 \\
1.582230 \\
1.581992 \\
1.581755\end{array}$ & $\begin{array}{l}\text { 1. } 582677 \\
\text { 1. } 582434 \\
1.582193 \\
1.581955 \\
1.581718\end{array}$ & $\begin{array}{l}\text { 1. } 582639 \\
1.582396 \\
1.582155 \\
1.581917 \\
1.581680\end{array}$ \\
\hline $\begin{array}{l}4350 \text { - } \\
4360_{-} \\
4370_{-} \\
4380_{-} \\
4390_{-}\end{array}$ & $\begin{array}{l}1.581895 \\
1.581662 \\
1.581431 \\
1.581201 \\
1.580974\end{array}$ & $\begin{array}{l}\text { 1. } 581857 \\
\text { 1. } 581625 \\
\text { 1. } 581394 \\
\text { 1. } 581164 \\
1.580937\end{array}$ & $\begin{array}{l}1.581820 \\
1.581587 \\
1.581356 \\
1.581126 \\
1.580899\end{array}$ & $\begin{array}{l}1.581319 \\
1.581089 \\
1.580862\end{array}$ & & & $\begin{array}{l}\text { 1. } 581670 \\
1.581437 \\
1.581206 \\
\text { 1. } 580976 \\
1.580749\end{array}$ & & $\begin{array}{l}1.581595 \\
1.581362 \\
1.581131 \\
1.580901 \\
1.580674\end{array}$ & $\begin{array}{l}1.581558 \\
1.581324 \\
1.581093 \\
1.580863 \\
1.580636\end{array}$ & $\begin{array}{l}1.581520 \\
1.581287 \\
1.581056 \\
1.580826 \\
1.580599\end{array}$ & $\begin{array}{l}1.581483 \\
1.581249 \\
1.581018 \\
1.580788 \\
1.580561\end{array}$ & $\begin{array}{l}\text { 1. } 581445 \\
\text { 1. } 581212 \\
\text { 1. } 580981 \\
\text { 1. } 580751 \\
1.580524\end{array}$ \\
\hline & $\begin{array}{l}\text { 1. } 580748 \\
1.580524 \\
1.580302 \\
1.580083 \\
1.579865\end{array}$ & $\begin{array}{l}\text { 1. } 580711 \\
\text { 1. } 580487 \\
\text { 1. } 580265 \\
\text { 1. } 580045 \\
1.579827\end{array}$ & $\begin{array}{l}\text { 1. } 580673 \\
\text { 1. } 580449 \\
\text { 1. } 580227 \\
1.580007 \\
1.579789\end{array}$ & $\begin{array}{l}1.580636 \\
1.580412 \\
1.580190 \\
1.579970 \\
1.579752\end{array}$ & $\begin{array}{l}1.580598 \\
1.580374 \\
1.580152 \\
1.579932 \\
1.579714\end{array}$ & $\begin{array}{l}1.580561 \\
1.580337 \\
1.580115 \\
1.579895 \\
1.579677\end{array}$ & $\begin{array}{l}\text { 1. } 580523 \\
\text { 1. } 580299 \\
\text { 1. } 580077 \\
\text { 1. } 579857 \\
1.579639\end{array}$ & $\begin{array}{l}1.580485 \\
1.580261 \\
1.580039 \\
1.579819 \\
1.579601\end{array}$ & $\begin{array}{l}\text { 1. } 580448 \\
\text { 1. } 580224 \\
\text { 1. } 580002 \\
\text { 1. } 579782 \\
1.579564\end{array}$ & $\begin{array}{l}\text { 1. } 580410 \\
\text { 1. } 580186 \\
\text { 1. } 579964 \\
1.579744 \\
1.579526\end{array}$ & $\begin{array}{l}1.580373 \\
1.580149 \\
1.579927 \\
1.579707 \\
1.579489\end{array}$ & $\begin{array}{l}\text { 1. } 580335 \\
\text { 1. } 580111 \\
1.579889 \\
1.579669 \\
1.579451\end{array}$ & $\begin{array}{l}\text { 1. } 580298 \\
\text { 1. } 580074 \\
1.579852 \\
1.579631 \\
1.579413\end{array}$ \\
\hline $\begin{array}{l}4450_{-} \\
4460_{-} \\
4470- \\
4480_{-} \\
4490_{-}\end{array}$ & $\begin{array}{l}1.579648 \\
1.579433 \\
1.579219 \\
1.579008 \\
1.578798\end{array}$ & $\begin{array}{l}1.579610 \\
1.579395 \\
1.579181 \\
1.578970 \\
1.578760\end{array}$ & $\begin{array}{l}1.579572 \\
1.579358 \\
1.579144 \\
1.578933 \\
1.578723\end{array}$ & $\begin{array}{l}1.579320 \\
1.579106 \\
1.578895 \\
1.578685\end{array}$ & $\begin{array}{l}\text { 1. } 579068 \\
\text { 1. } 578857 \\
\text { 1. } 578647\end{array}$ & $\begin{array}{l}1.578820 \\
1.578610\end{array}$ & $\begin{array}{l}1.579422 \\
1.579207 \\
1.578993 \\
1.578782 \\
1.578572\end{array}$ & $\begin{array}{l}1.579584 \\
1.579169 \\
1.578955 \\
1.578744 \\
1.578534\end{array}$ & $\begin{array}{l}1.579347 \\
1.579132 \\
1.578918 \\
1.578707 \\
1.578497\end{array}$ & & $\begin{array}{l}1.579272 \\
1.579056 \\
1.578842 \\
1.578631 \\
1.578421\end{array}$ & $\begin{array}{l}1.579234 \\
1.579019 \\
1.578805 \\
1.578594 \\
1.578384\end{array}$ & $\begin{array}{l}\text { 1. } 579196 \\
\text { 1. } 578981 \\
1.578767 \\
1.578556 \\
1.578346\end{array}$ \\
\hline & $\begin{array}{l}1.578589 \\
1.578382 \\
1.578177 \\
1.577973 \\
1.577771\end{array}$ & $\begin{array}{l}\text { 1. } 578551 \\
\text { 1. } 578344 \\
1.578140 \\
1.577936 \\
1.577734\end{array}$ & $\begin{array}{l}1.578514 \\
1.578307 \\
1.578102 \\
1.577898 \\
1.577696\end{array}$ & $\begin{array}{l}1.578476 \\
1.578269 \\
1.578064 \\
1.577860 \\
1.577658\end{array}$ & $\begin{array}{l}1.578438 \\
1.578231 \\
1.578026 \\
1.577822 \\
1.577620\end{array}$ & $\begin{array}{l}1.577194 \\
1.577989 \\
1.577785 \\
1.577583\end{array}$ & $\begin{array}{l}\text { 1. } 578363 \\
\text { 1. } 578156 \\
\text { 1. } 577951 \\
\text { 1. } 577747 \\
1.577545\end{array}$ & $\begin{array}{l}1.578118 \\
1.577913 \\
1.577709 \\
1.577507\end{array}$ & $\begin{array}{l}1.578288 \\
1.578081 \\
1.577876 \\
1.577672 \\
1.577470\end{array}$ & $\begin{array}{l}1.578250 \\
1.578043 \\
1.577838 \\
1.577634 \\
1.577432\end{array}$ & $\begin{array}{l}1.578212 \\
1.578005 \\
1.577800 \\
1.577596 \\
1.577394\end{array}$ & $\begin{array}{l}1.578175 \\
1.577968 \\
1.577762 \\
1.577558 \\
1.577356\end{array}$ & $\begin{array}{l}\text { 1. } 578137 \\
1.577930 \\
1.577725 \\
1.577521 \\
1.577319\end{array}$ \\
\hline $\begin{array}{l}4550 \\
4560 \\
4570 \\
4580 \\
4590\end{array}$ & $\begin{array}{l}1.577571 \\
1.577372 \\
1.577175 \\
1.576979 \\
1.576784\end{array}$ & $\begin{array}{l}1.577533 \\
1.577334 \\
1.577137 \\
1.576941 \\
1.576746\end{array}$ & $\begin{array}{l}1.577495 \\
1.577296 \\
1.577099 \\
1.576903 \\
1.576708\end{array}$ & $\begin{array}{l}1.577457 \\
1.577258 \\
1.577061 \\
1.576865 \\
1.576670\end{array}$ & $\begin{array}{l}1.577420 \\
1.577221 \\
1.577024 \\
1.576828 \\
1.576633\end{array}$ & $\begin{array}{l}1.576790 \\
1.576595\end{array}$ & $\begin{array}{l}1.577344 \\
1.577145 \\
1.576948 \\
1.576752 \\
1.576557\end{array}$ & $\begin{array}{l}1.56710 \\
1.576714 \\
1.576519\end{array}$ & $\begin{array}{l}1.577268 \\
1.577069 \\
1.576872 \\
1.576676 \\
1.576481\end{array}$ & $\begin{array}{l}1.577231 \\
1.577032 \\
1.576835 \\
1.576639 \\
1.576444\end{array}$ & $\begin{array}{l}1.577193 \\
1.576994 \\
1.576797 \\
1.576601 \\
1.576406\end{array}$ & $\begin{array}{l}1.577155 \\
1.576956 \\
1.576759 \\
1.576563 \\
1.576368\end{array}$ & $\begin{array}{l}1.577117 \\
1.576918 \\
1.576721 \\
1.576525 \\
1.576330\end{array}$ \\
\hline & $\begin{array}{l}1.576591 \\
1.576399 \\
1.576209 \\
1.576020 \\
1.575833\end{array}$ & $\begin{array}{l}1.576553 \\
1.576361 \\
1.576171 \\
1.575982 \\
1.575795\end{array}$ & $\begin{array}{l}1.576515 \\
1.576323 \\
1.576133 \\
1.575944 \\
1.575757\end{array}$ & $\begin{array}{l}1.576280 \\
1.576096 \\
1.575907 \\
1.575720\end{array}$ & $\begin{array}{l}1.576248 \\
1.576058 \\
1.575689 \\
1.575682\end{array}$ & $\begin{array}{l}1.576020 \\
1.575831 \\
1.575644\end{array}$ & $\begin{array}{l}\text { 1. } 576172 \\
1.575982 \\
1.575793 \\
1.575606\end{array}$ & $\begin{array}{l}1.576134 \\
1.575944 \\
1.575755 \\
1.575568\end{array}$ & $\begin{array}{l}1.576288 \\
1.576096 \\
1.575906 \\
1.575717 \\
1.575530\end{array}$ & $\begin{array}{l}1.576251 \\
1.576059 \\
1.575868 \\
1.575679 \\
1.575492\end{array}$ & $\begin{array}{l}1.576021 \\
1.575831 \\
1.575642 \\
1.575455\end{array}$ & $\begin{array}{l}1.576175 \\
1.575983 \\
1.575793 \\
1.575604 \\
1.575417\end{array}$ & $\begin{array}{l}1.575945 \\
1.575755 \\
1.575566 \\
1.575379\end{array}$ \\
\hline $\begin{array}{l}4650- \\
4660- \\
4670- \\
4680^{-} \\
4690_{-}\end{array}$ & $\begin{array}{l}1.575647 \\
1.575462 \\
1.575279 \\
1.575098 \\
1.574917\end{array}$ & $\begin{array}{l}1.575424 \\
\text { 1. } 575242 \\
1.575060 \\
1.574879\end{array}$ & $\begin{array}{l}1.575387 \\
1.575204 \\
1.575022 \\
1.574841\end{array}$ & $\begin{array}{l}1.575534 \\
1.575349 \\
1.575166 \\
1.574984 \\
1.574803\end{array}$ & $\begin{array}{l}1.575496 \\
1.575311 \\
1.575128 \\
1.574946 \\
1.574765\end{array}$ & $\begin{array}{l}1.575458 \\
1.575273 \\
1.575090 \\
1.574908 \\
1.574727\end{array}$ & $\begin{array}{l}1.575420 \\
1.575235 \\
1.575052 \\
1.574870 \\
1.574689\end{array}$ & $\begin{array}{l}1.575382 \\
1.575197 \\
1.575014 \\
1.574832 \\
1.574651\end{array}$ & $\begin{array}{l}1.575344 \\
1.575159 \\
1.574976 \\
1.574794 \\
1.574613\end{array}$ & $\begin{array}{l}1.575306 \\
1.575121 \\
1.574938 \\
1.574756 \\
1.574575\end{array}$ & $\begin{array}{l}1.575268 \\
1.575083 \\
1.574900 \\
1.574718 \\
1.574537\end{array}$ & $\begin{array}{l}1.575231 \\
1.575046 \\
1.574862 \\
1.574680 \\
1.574499\end{array}$ & $\begin{array}{l}1.575193 \\
1.575008 \\
1.574825 \\
1.574642 \\
1.574461\end{array}$ \\
\hline $\begin{array}{l}4700 \\
4710 \\
4720 \\
4730 \\
4740\end{array}$ & $\begin{array}{l}1.574738 \\
1.574560 \\
1.574383 \\
1.574208 \\
1.574034\end{array}$ & $\begin{array}{l}\text { 1. } 574700 \\
\text { 1. } 574522 \\
\text { 1. } 574345 \\
\text { 1. } 574170 \\
1.573996\end{array}$ & $\begin{array}{l}\text { 1. } 574662 \\
\text { 1. } 574484 \\
\text { 1. } 574307 \\
1.574132 \\
1.573958\end{array}$ & $\begin{array}{l}1.574624 \\
1.574446 \\
1.574269 \\
1.574094 \\
1.573920\end{array}$ & $\begin{array}{l}\text { 1. } 574408 \\
\text { 1. } 574231 \\
1.574056 \\
1.573882\end{array}$ & $\begin{array}{l}1.574370 \\
1.574193 \\
1.574018 \\
1.573844\end{array}$ & $\begin{array}{l}\text { 1. } 5743332 \\
\text { 1. } 574155 \\
1.573980 \\
1.573806\end{array}$ & $\begin{array}{l}1.574294 \\
1.574117 \\
1.573942 \\
1.573768\end{array}$ & $\begin{array}{l}\text { 1. } 574256 \\
\text { 1. } 574079 \\
1.573904 \\
1.573730\end{array}$ & $\begin{array}{l}1.574396 \\
1.574218 \\
1.574041 \\
1.573866 \\
1.573692\end{array}$ & $\begin{array}{l}1.574358 \\
1.574180 \\
1.574003 \\
1.573828 \\
1.573654\end{array}$ & $\begin{array}{l}1.574320 \\
1.574142 \\
1.573965 \\
1.573790 \\
1.573616\end{array}$ & $\begin{array}{l}\text { 1. } 574104 \\
1.573927 \\
1.573752 \\
1.573578\end{array}$ \\
\hline
\end{tabular}


TавLE 1. Refractive indices of potassium bromide-Continued

[Computed from equations 1 and 2]

\begin{tabular}{|c|c|c|c|c|c|c|c|c|c|c|c|c|c|}
\hline \multirow{2}{*}{ Wavelength } & \multicolumn{13}{|c|}{ Refractive index at - } \\
\hline & $16^{\circ} \mathrm{C}$ & $17^{\circ} \mathrm{C}$ & $18^{\circ} \mathrm{C}$ & $19^{\circ} \mathrm{C}$ & $20^{\circ} \mathrm{C}$ & $21^{\circ} \mathrm{C}$ & $22^{\circ} \mathrm{C}$ & $23^{\circ} \mathrm{C}$ & $24^{\circ} \mathrm{C}$ & $25^{\circ} \mathrm{C}$ & $26^{\circ} \mathrm{C}$ & $27^{\circ} \mathrm{C}$ & $28^{\circ} \mathrm{C}$ \\
\hline $\begin{array}{l}4750 \ldots \\
4760_{-} \\
4770_{-} \\
4780_{-} \\
4790_{-}\end{array}$ & $\begin{array}{l}1.573861 \\
1.573689 \\
1.573519 \\
1.573350 \\
1.573182\end{array}$ & $\begin{array}{l}1.573823 \\
1.573651 \\
1.573481 \\
1.573312 \\
1.573144\end{array}$ & $\begin{array}{l}\text { 1. } 573785 \\
1.573613 \\
1.573443 \\
1.573274 \\
1.573106\end{array}$ & $\begin{array}{l}1.573747 \\
1.573575 \\
1.573405 \\
1.573236 \\
1.573068\end{array}$ & $\begin{array}{l}1.573709 \\
1.573537 \\
1.573367 \\
1.573198 \\
1.573030\end{array}$ & $\begin{array}{l}1.573671 \\
1.573499 \\
1.573329 \\
1.573160 \\
1.572992\end{array}$ & $\begin{array}{l}1.573633 \\
1.573461 \\
1.573291 \\
1.573122 \\
1.572954\end{array}$ & $\begin{array}{l}1.573595 \\
1.573423 \\
1.573253 \\
1.573084 \\
1.572916\end{array}$ & $\begin{array}{l}1.573557 \\
1.573385 \\
1.573215 \\
1.573046 \\
1.572878\end{array}$ & $\begin{array}{l}1.573519 \\
1.573347 \\
1.573177 \\
1.573008 \\
1.572840\end{array}$ & $\begin{array}{l}1.573481 \\
1.573309 \\
1.573139 \\
1.572970 \\
1.572802\end{array}$ & $\begin{array}{l}\text { 1. } 573443 \\
\text { 1. } 573271 \\
\text { 1. } 573101 \\
\text { 1. } 572932 \\
1.572764\end{array}$ & $\begin{array}{l}1.573405 \\
1.573233 \\
1.573063 \\
1.572894 \\
1.572725\end{array}$ \\
\hline $\begin{array}{l}4800_{\ldots} \\
4810_{\ldots} \\
4820 \ldots \\
4830_{\ldots} \\
4840_{\ldots}\end{array}$ & $\begin{array}{l}\text { 1. } 573015 \\
1.572850 \\
1.572686 \\
1.572522 \\
1.572360\end{array}$ & $\begin{array}{l}1.572977 \\
1.572811 \\
1.572648 \\
1.572484 \\
1.572322\end{array}$ & $\begin{array}{l}1.572939 \\
1.572773 \\
1.572609 \\
1.572445 \\
1.572284\end{array}$ & $\begin{array}{l}\text { 1. } 572901 \\
1.572735 \\
1.572571 \\
1.572407 \\
1.572245\end{array}$ & $\begin{array}{l}\text { 1. } 572863 \\
\text { 1. } 572697 \\
1.572533 \\
\text { 1. } 572369 \\
1.572207\end{array}$ & $\begin{array}{l}\text { 1. } 572825 \\
\text { 1. } 572659 \\
1.572495 \\
\text { 1. } 572331 \\
1.572169\end{array}$ & $\begin{array}{l}\text { 1. } 572787 \\
\text { 1. } 572621 \\
\text { 1. } 572457 \\
\text { 1. } 572293 \\
\text { 1. } 572131\end{array}$ & $\begin{array}{l}\text { 1. } 572749 \\
1.572583 \\
\text { 1. } 572419 \\
\text { 1. } 572255 \\
\text { 1. } 572093\end{array}$ & $\begin{array}{l}1.572711 \\
1.572545 \\
1.572381 \\
1.572217 \\
1.572055\end{array}$ & $\begin{array}{l}1.572673 \\
1.572507 \\
1.572343 \\
1.572179 \\
1.572017\end{array}$ & $\begin{array}{l}\text { 1. } 572635 \\
\text { 1. } 572469 \\
\text { 1. } 572305 \\
\text { 1. } 572141 \\
1.571978\end{array}$ & $\begin{array}{l}\text { 1. } 572597 \\
\text { 1. } 572430 \\
\text { 1. } 572266 \\
\text { 1. } 572102 \\
1.571940\end{array}$ & $\begin{array}{l}\text { 1. } 572559 \\
\text { 1. } 572392 \\
\text { 1. } 572228 \\
\text { 1. } 572064 \\
\text { 1. } 571902\end{array}$ \\
\hline $\begin{array}{l}4850 \ldots \\
4860_{\ldots} \\
4870 \\
488 \\
4890_{-}\end{array}$ & $\begin{array}{l}\text { 1. } 572199 \\
\text { 1. } 572039 \\
1.571881 \\
1.571723 \\
1.571567\end{array}$ & $\begin{array}{l}\text { 1. } 572161 \\
\text { 1. } 572001 \\
1.571843 \\
1.571685 \\
1.571529\end{array}$ & $\begin{array}{l}1.572123 \\
1.571963 \\
1.571805 \\
1.571647 \\
1.571491\end{array}$ & $\begin{array}{l}\text { 1. } 572084 \\
1.571924 \\
1.571767 \\
1.571609 \\
1.571453\end{array}$ & $\begin{array}{l}1.572046 \\
1.571886 \\
1.571728 \\
1.571570 \\
1.571414\end{array}$ & $\begin{array}{l}\text { 1. } 572008 \\
\text { 1. } 571848 \\
\text { 1. } 571690 \\
\text { 1. } 571532 \\
1.571376\end{array}$ & $\begin{array}{l}\text { 1. } 571970 \\
\text { 1. } 571810 \\
\text { 1. } 571652 \\
\text { 1. } 571494 \\
1.571338\end{array}$ & $\begin{array}{l}1.571932 \\
1.571772 \\
1.571614 \\
1.571456 \\
1.571300\end{array}$ & $\begin{array}{l}1.571894 \\
1.571734 \\
1.571576 \\
1.571418 \\
1.571261\end{array}$ & $\begin{array}{l}1.571856 \\
1.571696 \\
1.571537 \\
1.571379 \\
1.571223\end{array}$ & $\begin{array}{l}1.571817 \\
\text { 1. } 571657 \\
\text { 1. } 571499 \\
\text { 1. } 571341 \\
1.571185\end{array}$ & $\begin{array}{l}\text { 1. } 571779 \\
1.571619 \\
1.571461 \\
1.571303 \\
1.571147\end{array}$ & $\begin{array}{l}1.571741 \\
1.571581 \\
1.571423 \\
1.571265 \\
1.571109\end{array}$ \\
\hline $\begin{array}{l}4900-- \\
4910= \\
4920= \\
4930= \\
4940=\end{array}$ & $\begin{array}{l}\text { 1. } 571411 \\
1.571257 \\
\text { 1. } 571103 \\
1.570952 \\
1.570801\end{array}$ & $\begin{array}{l}\text { 1. } 571373 \\
\text { 1. } 571219 \\
1.571065 \\
1.570913 \\
1.570762\end{array}$ & $\begin{array}{l}\text { 1. } 571335 \\
\text { 1. } 571181 \\
\text { 1. } 571027 \\
1.570875 \\
1.570724\end{array}$ & $\begin{array}{l}\text { 1. } 571297 \\
1.571143 \\
1.570989 \\
1.570837 \\
1.570686\end{array}$ & $\begin{array}{l}1.571258 \\
1.571104 \\
1.570950 \\
1.570799 \\
1.570647\end{array}$ & $\begin{array}{l}\text { 1. } 571220 \\
\text { 1. } 571066 \\
\text { 1. } 570912 \\
\text { 1. } 570760 \\
\text { 1. } 570609\end{array}$ & $\begin{array}{l}\text { 1. } 571182 \\
\text { 1. } 571028 \\
\text { 1. } 570874 \\
\text { 1. } 570722 \\
\text { 1. } 570571\end{array}$ & $\begin{array}{l}\text { 1. } 571144 \\
\text { 1. } 570990 \\
\text { 1. } 570836 \\
\text { 1. } 570684 \\
1.570533\end{array}$ & $\begin{array}{l}1.571106 \\
1.570951 \\
1.570798 \\
1.570645 \\
1.570494\end{array}$ & $\begin{array}{l}\text { 1. } 571067 \\
1.570913 \\
1.570759 \\
1.570607 \\
1.570456\end{array}$ & $\begin{array}{l}\text { 1. } 571029 \\
\text { 1. } 570875 \\
\text { 1. } 570721 \\
\text { 1. } 570569 \\
\text { 1. } 570418\end{array}$ & $\begin{array}{l}\text { 1. } 570991 \\
\text { 1. } 570837 \\
\text { 1. } 570683 \\
\text { 1. } 570531 \\
\text { 1. } 570380\end{array}$ & $\begin{array}{l}\text { 1. } 570953 \\
\text { 1. } 570799 \\
\text { 1. } 570645 \\
\text { 1. } 570492 \\
\text { 1. } 570341\end{array}$ \\
\hline $\begin{array}{l}4950 . \\
4960 . \\
4970- \\
4980_{-} \\
4990_{-}\end{array}$ & $\begin{array}{l}\text { 1. } 570651 \\
1.570502 \\
1.570353 \\
1.570206 \\
1.570060\end{array}$ & $\begin{array}{l}\text { 1. } 570612 \\
1.570463 \\
1.570315 \\
1.570168 \\
1.570022\end{array}$ & $\begin{array}{l}\text { 1. } 570574 \\
\text { 1. } 570425 \\
\text { 1. } 570276 \\
\text { 1. } 570129 \\
\text { 1. } 569983\end{array}$ & $\begin{array}{l}\text { 1. } 570536 \\
1.570387 \\
1.570238 \\
1.570091 \\
1.569945\end{array}$ & $\begin{array}{l}1.570498 \\
1.570349 \\
1.570200 \\
1.570053 \\
1.569907\end{array}$ & $\begin{array}{l}\text { 1. } 570459 \\
\text { 1. } 570310 \\
\text { 1. } 570161 \\
\text { 1. } 570014 \\
\text { 1. } 569868\end{array}$ & $\begin{array}{l}\text { 1. } 570421 \\
\text { 1. } 570272 \\
\text { 1. } 570123 \\
\text { 1. } 569976 \\
1.569830\end{array}$ & $\begin{array}{l}\text { 1. } 570383 \\
\text { 1. } 570234 \\
\text { 1. } 570085 \\
\text { 1. } 569938 \\
1.569792\end{array}$ & $\begin{array}{l}\text { 1. } 570344 \\
\text { 1. } 570195 \\
1.570046 \\
1.569899 \\
1.569753\end{array}$ & $\begin{array}{l}1.570306 \\
1.570157 \\
1.570008 \\
1.569861 \\
1.569715\end{array}$ & $\begin{array}{l}\text { 1. } 570268 \\
\text { 1. } 570119 \\
\text { 1. } 569970 \\
\text { 1. } 569823 \\
1.569677\end{array}$ & $\begin{array}{l}\text { 1. } 570230 \\
\text { 1. } 570081 \\
\text { 1. } 569931 \\
\text { 1. } 569784 \\
\text { 1. } 569638\end{array}$ & $\begin{array}{l}\text { 1. } 570191 \\
\text { 1. } 570042 \\
\text { 1. } 569893 \\
1.569746 \\
1.569600\end{array}$ \\
\hline $\begin{array}{l}5000 \ldots \\
5010 \\
5020 \\
5000 \\
5040\end{array}$ & $\begin{array}{l}\text { 1. } 569915 \\
\text { 1. } 569771 \\
\text { 1. } 569628 \\
1.569486 \\
1.569344\end{array}$ & $\begin{array}{l}\text { 1. } 569877 \\
1.569733 \\
1.569590 \\
1.569448 \\
1.569306\end{array}$ & $\begin{array}{l}\text { 1. } 569838 \\
1.569694 \\
1.569551 \\
1.569410 \\
1.569267\end{array}$ & $\begin{array}{l}1.569800 \\
1.569656 \\
1.569513 \\
1.569371 \\
1.569229\end{array}$ & $\begin{array}{l}1.569762 \\
1.569618 \\
1.569475 \\
1.569333 \\
1.569191\end{array}$ & $\begin{array}{l}\text { 1. } 569723 \\
\text { 1. } 569579 \\
\text { 1. } 569436 \\
\text { 1. } 569294 \\
\text { 1. } 569152\end{array}$ & $\begin{array}{l}\text { 1. } 569685 \\
\text { 1. } 569541 \\
\text { 1. } 569398 \\
1.569256 \\
1.569114\end{array}$ & $\begin{array}{l}\text { 1. } 569647 \\
\text { 1. } 569503 \\
1.569360 \\
1.569218 \\
1.569076\end{array}$ & $\begin{array}{l}\text { 1. } 569608 \\
\text { 1. } 569464 \\
\text { 1. } 569321 \\
1.569179 \\
1.569037\end{array}$ & $\begin{array}{l}\text { 1. } 569570 \\
1.569426 \\
1.569283 \\
1.569141 \\
1.568999\end{array}$ & $\begin{array}{l}\text { 1. } 569532 \\
\text { 1. } 569387 \\
\text { 1. } 569245 \\
\text { 1. } 569102 \\
\text { 1. } 568960\end{array}$ & & $\begin{array}{l}\text { 1. } 569455 \\
\text { 1. } 569311 \\
\text { 1. } 569168 \\
\text { 1. } 569026 \\
\text { 1. } 568884\end{array}$ \\
\hline $\begin{array}{l}5050 \\
5060 \\
5070 \\
5080 \\
5090\end{array}$ & $\begin{array}{l}1.569204 \\
1.569066 \\
1.568927 \\
1.568790 \\
1.568653\end{array}$ & $\begin{array}{l}1.569166 \\
1.569027 \\
1.568888 \\
1.568751 \\
1.568614\end{array}$ & $\begin{array}{l}\text { 1. } 569128 \\
1.568989 \\
1.568850 \\
1.568713 \\
1.568576\end{array}$ & $\begin{array}{l}1.569089 \\
1.568950 \\
1.568811 \\
1.568674 \\
1.568537\end{array}$ & $\begin{array}{l}1.569051 \\
1.568912 \\
1.568773 \\
1.568636 \\
1.568499\end{array}$ & $\begin{array}{l}\text { 1. } 569012 \\
\text { 1. } 568873 \\
\text { 1. } 568734 \\
\text { 1. } 568597 \\
\text { 1. } 568460\end{array}$ & $\begin{array}{l}\text { 1. } 568974 \\
1.568835 \\
1.568696 \\
1.568559 \\
1.568422\end{array}$ & $\begin{array}{l}\text { 1. } 568936 \\
1.568797 \\
1.568658 \\
1.568521 \\
1.568383\end{array}$ & $\begin{array}{l}\text { 1. } 568897 \\
\text { 1. } 568758 \\
\text { 1. } 568619 \\
\text { 1. } 568482 \\
1.568345\end{array}$ & $\begin{array}{l}1.568859 \\
1.568720 \\
1.568581 \\
1.568444 \\
1.568307\end{array}$ & $\begin{array}{l}\text { 1. } 568820 \\
1.568681 \\
\text { 1. } 568542 \\
\text { 1. } 568405 \\
1.568268\end{array}$ & $\begin{array}{l}\text { 1. } 568782 \\
\text { 1. } 568643 \\
\text { 1. } 568504 \\
\text { 1. } 568367 \\
1.568230\end{array}$ & $\begin{array}{l}1.568744 \\
1.568604 \\
1.568465 \\
1.568328 \\
1.568191\end{array}$ \\
\hline $\begin{array}{l}5100 \\
5110- \\
5120_{-} \\
5130_{-} \\
5140_{-}\end{array}$ & $\begin{array}{l}\text { 1. } 568517 \\
\text { 1. } 568382 \\
\text { 1. } 568248 \\
1.568115 \\
1.567983\end{array}$ & $\begin{array}{l}1.568478 \\
1.568343 \\
1.568210 \\
1.568077 \\
1.567945\end{array}$ & $\begin{array}{l}\text { 1. } 568440 \\
1.568305 \\
1.568171 \\
1.568038 \\
1.567906\end{array}$ & $\begin{array}{l}\text { 1. } 568401 \\
1.568266 \\
1.568133 \\
1.568000 \\
1.567867\end{array}$ & $\begin{array}{l}1.568363 \\
1.568228 \\
1.568094 \\
1.567961 \\
1.567829\end{array}$ & $\begin{array}{l}\text { 1. } 568324 \\
\text { 1. } 568189 \\
\text { 1. } 568056 \\
\text { 1. } 567923 \\
1.567791\end{array}$ & $\begin{array}{l}\text { 1. } 568286 \\
\text { 1. } 568151 \\
\text { 1. } 568017 \\
\text { 1. } 567884 \\
\text { 1. } 567752\end{array}$ & & & & & $\begin{array}{l}\text { 1. } 568094 \\
\text { 1. } 567959 \\
\text { 1. } 567824 \\
\text { 1. } 567691 \\
\text { 1. } 567559\end{array}$ & $\begin{array}{l}\text { 1. } 568055 \\
\text { 1. } 567920 \\
\text { 1. } 567786 \\
\text { 1. } 567653 \\
\text { 1. } 567521\end{array}$ \\
\hline & $\begin{array}{l}\text { 1. } 567852 \\
\text { 1. } 567721 \\
\text { 1. } 567592 \\
1.567464 \\
1.567335\end{array}$ & $\begin{array}{l}1.567814 \\
1.567683 \\
1.567554 \\
1.567425 \\
1.567297\end{array}$ & $\begin{array}{l}1.567775 \\
1.567644 \\
1.567515 \\
1.567386 \\
1.567258\end{array}$ & $\begin{array}{l}\text { 1. } 567737 \\
1.567606 \\
1.567477 \\
1.567348 \\
1.567220\end{array}$ & $\begin{array}{l}1.567698 \\
1.567567 \\
1.567438 \\
1.567309 \\
1.567181\end{array}$ & $\begin{array}{l}\text { 1. } 567660 \\
\text { 1. } 567529 \\
\text { 1. } 567400 \\
\text { 1. } 567271 \\
1.567143\end{array}$ & $\begin{array}{l}\text { 1. } 567621 \\
1.567490 \\
1.567361 \\
1.567232 \\
1.567104\end{array}$ & $\begin{array}{l}\text { 1. } 567582 \\
\text { 1. } 567451 \\
\text { 1. } 567322 \\
\text { 1. } 567193 \\
1.567065\end{array}$ & $\begin{array}{l}1.567544 \\
1.567413 \\
1.567284 \\
1.567155 \\
1.567027\end{array}$ & $\begin{array}{l}1.567505 \\
1.567374 \\
1.567245 \\
1.567116 \\
1.566988\end{array}$ & $\begin{array}{l}\text { 1. } 567467 \\
\text { 1. } 567336 \\
\text { 1. } 567207 \\
\text { 1. } 567078 \\
\text { 1. } 566950\end{array}$ & $\begin{array}{l}\text { 1. } 567428 \\
\text { 1. } 567297 \\
\text { 1. } 567168 \\
\text { 1. } 567039 \\
1.566911\end{array}$ & $\begin{array}{l}1.567390 \\
1.567259 \\
1.567130 \\
1.567000 \\
1.566872\end{array}$ \\
\hline $\begin{array}{l}5200 \ldots \\
5210 \\
5220 \ldots \\
5230 \\
5240\end{array}$ & $\begin{array}{l}\text { 1. } 567209 \\
\text { 1. } 567082 \\
\text { 1. } 566957 \\
\text { 1. } 566832 \\
\text { 1. } 566708\end{array}$ & $\begin{array}{l}\text { 1. } 567170 \\
1.567043 \\
1.566918 \\
1.566793 \\
1.566669\end{array}$ & $\begin{array}{l}\text { 1. } 567131 \\
1.567004 \\
1.566880 \\
1.566755 \\
1.566631\end{array}$ & $\begin{array}{l}1.567093 \\
1.566966 \\
1.566841 \\
1.566716 \\
1.566592\end{array}$ & $\begin{array}{l}1.567054 \\
1.566927 \\
1.566802 \\
1.566677 \\
1.566553\end{array}$ & $\begin{array}{l}\text { 1. } 567016 \\
1.566889 \\
1.566764 \\
1.566639 \\
1.566515\end{array}$ & $\begin{array}{l}1.566977 \\
1.566850 \\
1.566725 \\
1.566600 \\
1.566476\end{array}$ & $\begin{array}{l}\text { 1. } 566938 \\
\text { 1. } 566811 \\
\text { 1. } 566686 \\
\text { 1. } 566561 \\
\text { 1. } 566437\end{array}$ & $\begin{array}{l}1.566900 \\
1.566773 \\
1.566648 \\
1.566523 \\
1.566399\end{array}$ & $\begin{array}{l}1.566861 \\
1.566734 \\
1.566609 \\
1.566484 \\
1.566360\end{array}$ & $\begin{array}{l}\text { 1. } 566823 \\
\text { 1. } 566695 \\
1.566570 \\
1.566445 \\
1.566321\end{array}$ & $\begin{array}{l}\text { 1. } 566784 \\
\text { 1. } 566657 \\
1.566532 \\
1.566407 \\
1.566283\end{array}$ & $\begin{array}{l}\text { 1. } 566745 \\
\text { 1. } 566618 \\
\text { 1. } 566493 \\
\text { 1. } 566368 \\
\text { 1. } 566244\end{array}$ \\
\hline & $\begin{array}{l}\text { 1. } 566585 \\
\text { 1. } 566463 \\
1.566341 \\
1.566220 \\
1.566100\end{array}$ & $\begin{array}{l}\text { 1. } 566546 \\
1.566424 \\
1.566303 \\
1.566182 \\
1.566062\end{array}$ & $\begin{array}{l}\text { 1. } 566508 \\
1.566386 \\
1.566264 \\
1.566143 \\
1.566023\end{array}$ & $\begin{array}{l}\text { 1. } 566469 \\
1.566347 \\
1.566225 \\
1.566104 \\
1.565984\end{array}$ & $\begin{array}{l}1.566430 \\
1.566308 \\
1.566186 \\
1.566065 \\
1.565945\end{array}$ & $\begin{array}{l}\text { 1. } 566392 \\
\text { 1. } 566270 \\
\text { 1. } 566148 \\
\text { 1. } 566027 \\
1.565907\end{array}$ & $\begin{array}{l}\text { 1. } 566353 \\
\text { 1. } 566231 \\
\text { 1. } 566109 \\
\text { 1. } 565988 \\
\text { 1. } 565868\end{array}$ & $\begin{array}{l}\text { 1. } 566314 \\
\text { 1. } 566192 \\
\text { 1. } 566070 \\
1.565949 \\
1.565829\end{array}$ & $\begin{array}{l}1.566276 \\
1.566154 \\
1.566032 \\
1.565911 \\
1.565791\end{array}$ & $\begin{array}{l}1.566237 \\
1.566115 \\
1.565993 \\
1.565872 \\
1.565752\end{array}$ & $\begin{array}{l}\text { 1. } 566198 \\
\text { 1. } 566076 \\
\text { 1. } 565954 \\
\text { 1. } 565833 \\
\text { 1. } 565713\end{array}$ & $\begin{array}{l}1.566160 \\
1.566038 \\
1.565915 \\
1.565794 \\
1.565674\end{array}$ & $\begin{array}{l}\text { 1. } 566121 \\
\text { 1. } 565999 \\
\text { 1. } 565877 \\
\text { 1. } 565756 \\
\text { 1. } 565636\end{array}$ \\
\hline $\begin{array}{l}5300 \ldots \\
5310_{-} \\
5320 \\
5330 \\
5340\end{array}$ & $\begin{array}{l}\text { 1. } 565981 \\
\text { 1. } 565863 \\
\text { 1. } 565745 \\
\text { 1. } 565628 \\
1.565512\end{array}$ & $\begin{array}{l}\text { 1. } 565943 \\
\text { 1. } 565824 \\
1.565706 \\
1.565589 \\
1.565473\end{array}$ & $\begin{array}{l}\text { 1. } 565904 \\
1.565785 \\
1.565667 \\
1.565550 \\
1.565434\end{array}$ & $\begin{array}{l}1.565865 \\
1.565746 \\
1.565828 \\
1.565511 \\
1.565395\end{array}$ & $\begin{array}{l}1.565826 \\
1.565707 \\
1.565590 \\
1.565473 \\
1.565357\end{array}$ & & $\begin{array}{l}1.565749 \\
1.565630 \\
1.565512 \\
1.565395 \\
1.565279\end{array}$ & & $\begin{array}{l}1.565672 \\
1.565552 \\
1.565434 \\
1.565317 \\
1.565201\end{array}$ & $\begin{array}{l}1.565633 \\
1.565514 \\
1.565396 \\
1.565279 \\
1.565163\end{array}$ & $\begin{array}{l}1.565594 \\
1.565475 \\
1.565357 \\
1.565240 \\
1.565124\end{array}$ & $\begin{array}{l}\text { 1. } 565555 \\
1.565436 \\
1.565318 \\
1.565201 \\
1.565085\end{array}$ & $\begin{array}{l}\text { 1. } 565517 \\
\text { 1. } 565397 \\
\text { 1. } 565279 \\
\text { 1. } 565162 \\
1.565046\end{array}$ \\
\hline & $\begin{array}{l}\text { 1. } 565396 \\
\text { 1. } 565282 \\
\text { 1. } 565167 \\
\text { 1. } 565054 \\
\text { 1. } 564941\end{array}$ & $\begin{array}{l}\text { 1. } 565357 \\
\text { 1. } 5652433 \\
1.565128 \\
1.565015 \\
1.564902\end{array}$ & $\begin{array}{l}\text { 1. } 565318 \\
1.565204 \\
1.565089 \\
1.564976 \\
1.564863\end{array}$ & $\begin{array}{l}1.565165 \\
1.565051 \\
1.564938 \\
1.564825\end{array}$ & $\begin{array}{l}1.565127 \\
1.565012 \\
1.564899 \\
1.564786\end{array}$ & $\begin{array}{l}\text { 1. } 565088 \\
\text { 1. } 564973 \\
1.564860 \\
1.564747\end{array}$ & $\begin{array}{l}1.565049 \\
1.564934 \\
1.564821 \\
1.564708\end{array}$ & $\begin{array}{l}1.565124 \\
1.565010 \\
1.564895 \\
1.564782 \\
1.564669\end{array}$ & $\begin{array}{l}1.565085 \\
1.564971 \\
1.564856 \\
1.564743 \\
1.564630\end{array}$ & $\begin{array}{l}1.565047 \\
1.564933 \\
1.564817 \\
1.564704 \\
1.564591\end{array}$ & $\begin{array}{l}\text { 1. } 565008 \\
\text { 1. } 564894 \\
\text { 1. } 564779 \\
\text { 1. } 564666 \\
\text { 1. } 564553\end{array}$ & $\begin{array}{l}1.564969 \\
1.564855 \\
1.564740 \\
1.564627 \\
1.564514\end{array}$ & $\begin{array}{l}\text { 1. } 564930 \\
\text { 1. } 564816 \\
\text { 1. } 564701 \\
\text { 1. } 564588 \\
\text { 1. } 564475\end{array}$ \\
\hline $\begin{array}{l}5400 \ldots \\
5410 \ldots \\
5420 \\
5430 \\
5440\end{array}$ & $\begin{array}{l}\text { 1. } 564829 \\
\text { 1. } 564718 \\
\text { 1. } 564607 \\
\text { 1. } 564497 \\
1.564388\end{array}$ & $\begin{array}{l}\text { 1. } 564790 \\
\text { 1. } 564679 \\
\text { 1. } 564569 \\
1.564459 \\
1.564349\end{array}$ & $\begin{array}{l}\text { 1. } 564752 \\
1.564641 \\
1.564530 \\
1.564420 \\
1.564310\end{array}$ & $\begin{array}{l}\text { 1. } 564713 \\
1.564602 \\
1.564491 \\
1.564381 \\
1.564271\end{array}$ & $\begin{array}{l}1.564674 \\
1.564563 \\
1.564452 \\
1.564342 \\
1.564232\end{array}$ & $\begin{array}{l}1.564635 \\
1.564524 \\
1.564413 \\
1.564303 \\
1.564193\end{array}$ & $\begin{array}{l}1.564596 \\
1.564485 \\
1.564374 \\
1.564264 \\
1.564154\end{array}$ & $\begin{array}{l}1.564557 \\
1.564446 \\
1.564335 \\
1.564225 \\
1.564115\end{array}$ & $\begin{array}{l}1.564518 \\
1.564407 \\
1.564296 \\
1.564186 \\
1.564076\end{array}$ & $\begin{array}{l}1.564479 \\
1.564368 \\
1.564257 \\
1.564147 \\
1.564037\end{array}$ & $\begin{array}{l}1.564441 \\
1.564329 \\
1.564218 \\
1.564108 \\
1.563998\end{array}$ & $\begin{array}{l}1.564402 \\
1.564291 \\
1.564179 \\
1.564069 \\
1.563959\end{array}$ & $\begin{array}{l}\text { 1. } 564363 \\
1.564252 \\
1.564141 \\
1.564031 \\
1.563920\end{array}$ \\
\hline $\begin{array}{l}5450 \ldots \\
5460 \ldots \\
5470-\ldots \\
5480 \ldots \\
5490 \ldots\end{array}$ & $\begin{array}{l}\text { 1. } 564172 \\
\text { 1. } 564064 \\
\text { 1. } 563958 \\
1.563851\end{array}$ & $\begin{array}{l}\text { 1. } 564241 \\
\text { 1. } 564133 \\
\text { 1. } 564025 \\
\text { 1. } 563919 \\
1.563812\end{array}$ & $\begin{array}{l}1.564094 \\
\text { 1. } 563986 \\
1.563880 \\
1.563773\end{array}$ & $\begin{array}{l}\text { 1. } 564055 \\
\text { 1. } 563947 \\
\text { 1. } 563841 \\
1.563734\end{array}$ & $\begin{array}{l}1.564016 \\
\text { 1. } 563908 \\
\text { 1. } 563802 \\
1.563695\end{array}$ & $\begin{array}{l}\text { 1. } 5639777 \\
\text { 1. } 563869 \\
\text { 1. } 563763 \\
1.563656\end{array}$ & $\begin{array}{l}1.564046 \\
1.563938 \\
1.563830 \\
1.563724 \\
1.563617\end{array}$ & $\begin{array}{l}1.564007 \\
1.563899 \\
1.563791 \\
1.563685 \\
1.563578\end{array}$ & $\begin{array}{l}1.563968 \\
1.563860 \\
1.563752 \\
1.563646 \\
1.563539\end{array}$ & $\begin{array}{l}1.563929 \\
1.563821 \\
1.563713 \\
1.563607 \\
1.563500\end{array}$ & $\begin{array}{l}1.563890 \\
1.563782 \\
1.563674 \\
1.563568 \\
1.563461\end{array}$ & $\begin{array}{l}1.563851 \\
1.563743 \\
1.563635 \\
1.563529 \\
1.563422\end{array}$ & $\begin{array}{l}1.563812 \\
1.563704 \\
1.563596 \\
1.563490 \\
1.563383\end{array}$ \\
\hline
\end{tabular}


TABLE 1. Refractive indices of potassium bromide-Continued

[Computed from equations 1 and 2]

\begin{tabular}{|c|c|c|c|c|c|c|c|c|c|c|c|c|c|}
\hline \multirow{2}{*}{ Wavelength } & \multicolumn{13}{|c|}{ Refractive index at - } \\
\hline & $16^{\circ} \mathrm{C}$ & $17^{\circ} \mathrm{C}$ & $18^{\circ} \mathrm{C}$ & $19^{\circ} \mathrm{C}$ & $20^{\circ} \mathrm{C}$ & $21^{\circ} \mathrm{C}$ & $22^{\circ} \mathrm{C}$ & $23^{\circ} \mathrm{C}$ & $24^{\circ} \mathrm{C}$ & $25^{\circ} \mathrm{C}$ & $26^{\circ} \mathrm{C}$ & $27^{\circ} \mathrm{C}$ & $28^{\circ} \mathrm{C}$ \\
\hline $\begin{array}{l}{ }^{A} \\
5500 \\
5520 \\
5540 \\
5580\end{array}$ & $\begin{array}{l}1.563746 \\
1.563537 \\
1.563330 \\
1.563127 \\
1.562925\end{array}$ & $\begin{array}{l}1.563707 \\
1.563498 \\
1.563291 \\
1.563087 \\
1.562886\end{array}$ & $\begin{array}{l}1.563668 \\
1.563459 \\
1.563252 \\
1.563048 \\
1.562846\end{array}$ & $\begin{array}{l}1.563629 \\
1.563420 \\
1.563213 \\
1.563009 \\
1.562807\end{array}$ & $\begin{array}{l}1.563590 \\
1.563381 \\
1.563174 \\
1.562970 \\
1.562768\end{array}$ & $\begin{array}{l}1.563551 \\
1.563342 \\
1.563135 \\
1.562931 \\
1.562729\end{array}$ & $\begin{array}{l}\text { 1. } 563512 \\
\text { 1. } 563303 \\
\text { 1. } 563096 \\
\text { 1. } 562892 \\
1.562690\end{array}$ & $\begin{array}{l}1.563473 \\
1.563264 \\
1.563057 \\
1.562853 \\
1.562651\end{array}$ & $\begin{array}{l}1.563434 \\
1.563225 \\
1.563018 \\
1.562814 \\
1.562612\end{array}$ & $\begin{array}{l}1.563395 \\
1.563186 \\
1.562979 \\
1.562775 \\
1.562573\end{array}$ & $\begin{array}{l}1.563356 \\
1.563147 \\
1.562940 \\
1.562736 \\
1.562534\end{array}$ & $\begin{array}{l}1.563317 \\
1.563108 \\
1.562901 \\
1.562697 \\
1.562494\end{array}$ & $\begin{array}{l}\text { 1. } 563278 \\
\text { 1. } 563069 \\
\text { 1. } 562862 \\
\text { 1. } 562657 \\
\text { 1. } 562455\end{array}$ \\
\hline $\begin{array}{l}5600 \\
5620 \\
5640 \\
5660 \\
5680\end{array}$ & $\begin{array}{l}1.562726 \\
1.562529 \\
1.562334 \\
1.562142 \\
1.561952\end{array}$ & $\begin{array}{l}\text { 1. } 562687 \\
1.562490 \\
1.562295 \\
1.562103 \\
1.561913\end{array}$ & $\begin{array}{l}\text { 1. } 562648 \\
1.562451 \\
1.562256 \\
1.562064 \\
1.561874\end{array}$ & $\begin{array}{l}\text { 1. } 562608 \\
1.562411 \\
1.562217 \\
1.562025 \\
1.561835\end{array}$ & $\begin{array}{l}1.562569 \\
1.562372 \\
1.562177 \\
1.561985 \\
1.561795\end{array}$ & $\begin{array}{l}1.562530 \\
1.562333 \\
1.562138 \\
1.561946 \\
1.561756\end{array}$ & $\begin{array}{l}\text { 1. } 562491 \\
1.562294 \\
1.562099 \\
1.561907 \\
1.561717\end{array}$ & $\begin{array}{l}1.562452 \\
1.562255 \\
1.562060 \\
1.561868 \\
1.561678\end{array}$ & $\begin{array}{l}1.562413 \\
1.562216 \\
1.562021 \\
1.561829 \\
1.561639\end{array}$ & $\begin{array}{l}\text { 1. } 562374 \\
\text { 1. } 562177 \\
1.561981 \\
1.561789 \\
1.561599\end{array}$ & $\begin{array}{l}1.562334 \\
1.562137 \\
1.561942 \\
1.561750 \\
1.561560\end{array}$ & $\begin{array}{l}1.562295 \\
1.562098 \\
1.561903 \\
1.561711 \\
1.561521\end{array}$ & $\begin{array}{l}\text { 1. } 562256 \\
\text { 1. } 562059 \\
\text { 1. } 561864 \\
\text { 1. } 561672 \\
\text { 1. } 561482\end{array}$ \\
\hline $\begin{array}{l}5700 \ldots \\
5720 \ldots \\
5740 \ldots \\
5760 \ldots \\
5780 \ldots\end{array}$ & $\begin{array}{l}\text { 1. } 561765 \\
\text { 1. } 561579 \\
\text { 1. } 561395 \\
\text { 1. } 561213 \\
1.561034\end{array}$ & $\begin{array}{l}1.561725 \\
1.561539 \\
1.561356 \\
1.561174 \\
1.560995\end{array}$ & $\begin{array}{l}1.561686 \\
1.561500 \\
1.561316 \\
1.561134 \\
1.560955\end{array}$ & $\begin{array}{l}\text { 1. } 561647 \\
\text { 1. } 561461 \\
1.561277 \\
1.561095 \\
1.560916\end{array}$ & $\begin{array}{l}\text { 1. } 561608 \\
1.561422 \\
1.561238 \\
1.561056 \\
1.560877\end{array}$ & $\begin{array}{l}\text { 1. } 561568 \\
1.561382 \\
1.561198 \\
1.561016 \\
1.560837\end{array}$ & $\begin{array}{l}\text { 1. } 561529 \\
1.561343 \\
1.561159 \\
1.560977 \\
1.560798\end{array}$ & $\begin{array}{l}1.561490 \\
1.561304 \\
1.561120 \\
1.560938 \\
1.560759\end{array}$ & $\begin{array}{l}1.561450 \\
1.561264 \\
1.561080 \\
1.560898 \\
1.560719\end{array}$ & $\begin{array}{l}\text { 1. } 561411 \\
1.561225 \\
1.561041 \\
1.560859 \\
1.560680\end{array}$ & $\begin{array}{l}1.561372 \\
1.561186 \\
1.561002 \\
1.560820 \\
1.560640\end{array}$ & $\begin{array}{l}1.561333 \\
1.561147 \\
1.560962 \\
1.560780 \\
1.560601\end{array}$ & $\begin{array}{l}\text { 1. } 561293 \\
\text { 1. } 561107 \\
\text { 1. } 560923 \\
\text { 1. } 560741 \\
1.560562\end{array}$ \\
\hline $\begin{array}{l}5800 \ldots \\
5820 \\
5840 \\
5860 \\
5880\end{array}$ & $\begin{array}{l}1.560856 \\
1.560681 \\
1.560508 \\
1.560336 \\
1.560166\end{array}$ & $\begin{array}{l}\text { 1. } 560817 \\
1.560641 \\
1.560468 \\
1.560296 \\
1.560127\end{array}$ & $\begin{array}{l}1.560778 \\
1.560602 \\
1.560429 \\
1.560257 \\
1.560087\end{array}$ & $\begin{array}{l}1.560738 \\
1.560562 \\
1.560389 \\
1.560217 \\
1.560047\end{array}$ & $\begin{array}{l}1.560699 \\
1.560523 \\
1.560350 \\
1.560178 \\
1.560008\end{array}$ & $\begin{array}{l}1.560659 \\
1.560483 \\
1.560310 \\
1.560138 \\
1.559969\end{array}$ & $\begin{array}{l}1.560620 \\
1.560444 \\
1.560271 \\
1.560099 \\
1.559929\end{array}$ & $\begin{array}{l}1.560581 \\
1.560405 \\
1.560232 \\
1.560060 \\
1.559889\end{array}$ & $\begin{array}{l}1.560541 \\
1.560365 \\
1.560192 \\
1.560020 \\
1.559850\end{array}$ & $\begin{array}{l}1.560502 \\
1.560326 \\
1.560153 \\
1.559981 \\
1.559810\end{array}$ & $\begin{array}{l}1.560462 \\
1.560286 \\
1.560113 \\
1.559941 \\
1.559771\end{array}$ & & $\begin{array}{l}1.560384 \\
1.560207 \\
1.560034 \\
1.559862 \\
1.559692\end{array}$ \\
\hline $\begin{array}{l}5900_{--} \\
5920_{--} \\
5940_{--} \\
5960- \\
5980-\end{array}$ & $\begin{array}{l}1.559998 \\
1.559832 \\
1.559669 \\
1.559506 \\
1.559346\end{array}$ & $\begin{array}{l}1.559959 \\
1.559793 \\
1.559629 \\
1.559466 \\
1.559306\end{array}$ & $\begin{array}{l}1.559919 \\
1.559753 \\
1.559589 \\
1.559426 \\
1.559267\end{array}$ & $\begin{array}{l}1.559880 \\
1.559714 \\
1.559550 \\
1.559387 \\
1.559227\end{array}$ & $\begin{array}{l}1.559840 \\
1.559674 \\
1.559510 \\
1.559347 \\
1.559187\end{array}$ & $\begin{array}{l}1.559801 \\
1.559635 \\
1.559471 \\
1.559308 \\
1.559148\end{array}$ & $\begin{array}{l}1.559761 \\
1.559595 \\
1.559431 \\
1.559268 \\
1.559108\end{array}$ & $\begin{array}{l}1.559721 \\
1.559555 \\
1.559391 \\
1.559228 \\
1.559068\end{array}$ & $\begin{array}{l}1.559682 \\
1.559516 \\
1.559352 \\
1.559189 \\
1.559029\end{array}$ & $\begin{array}{l}1.559642 \\
1.559476 \\
1.559312 \\
1.559149 \\
1.558989\end{array}$ & $\begin{array}{l}1.559603 \\
1.559437 \\
1.559273 \\
1.559110 \\
1.558949\end{array}$ & $\begin{array}{l}1.559563 \\
1.559397 \\
1.559233 \\
1.559070 \\
1.558910\end{array}$ & $\begin{array}{l}1.559524 \\
1.559358 \\
1.559193 \\
1.559030 \\
1.558870\end{array}$ \\
\hline $\begin{array}{l}6000 \\
6020 \\
6040 \\
6060 \\
6080\end{array}$ & $\begin{array}{l}1.559187 \\
\text { 1. } 559029 \\
1.558874 \\
1.558720 \\
1.558568\end{array}$ & $\begin{array}{l}1.559147 \\
1.558989 \\
1.558835 \\
1.558681 \\
1.558528\end{array}$ & $\begin{array}{l}1.559108 \\
1.558950 \\
1.558795 \\
1.558641 \\
1.558488\end{array}$ & $\begin{array}{l}1.559068 \\
1.558910 \\
1.558755 \\
1.558601 \\
1.558448\end{array}$ & $\begin{array}{l}1.559028 \\
1.558870 \\
1.558715 \\
1.558561 \\
1.558409\end{array}$ & $\begin{array}{l}\text { 1. } 558831 \\
\text { 1. } 558676 \\
1.558522 \\
1.558369\end{array}$ & $\begin{array}{l}\text { 1. } 558791 \\
\text { 1. } 558636 \\
\text { 1. } 558482 \\
\text { 1. } 558329\end{array}$ & $\begin{array}{l}1.558909 \\
1.558751 \\
1.558596 \\
1.558442 \\
1.558289\end{array}$ & $\begin{array}{l}1.558870 \\
1.558712 \\
1.558557 \\
1.558403 \\
1.558249\end{array}$ & $\begin{array}{l}1.558830 \\
1.558672 \\
1.558517 \\
1.558363 \\
1.558210\end{array}$ & $\begin{array}{l}1.558790 \\
1.558632 \\
1.558477 \\
1.558323 \\
1.558170\end{array}$ & $\begin{array}{l}1.558751 \\
1.558593 \\
1.558437 \\
1.558283 \\
1.558130\end{array}$ & $\begin{array}{l}1.558711 \\
1.558553 \\
1.558398 \\
1.558244 \\
1.558090\end{array}$ \\
\hline $\begin{array}{l}6100 \ldots \\
6120 \\
6140 \ldots \\
6160 \ldots \\
6180\end{array}$ & $\begin{array}{l}\text { 1. } 558418 \\
\text { 1. } 558269 \\
1.558121 \\
\text { 1. } 557975 \\
1.557831\end{array}$ & $\begin{array}{l}1.558378 \\
1.558229 \\
1.558081 \\
1.557935 \\
1.557791\end{array}$ & $\begin{array}{l}1.558338 \\
1.558189 \\
1.558041 \\
1.557896 \\
1.557752\end{array}$ & $\begin{array}{l}1.558298 \\
1.558149 \\
1.558002 \\
1.557856 \\
1.557712\end{array}$ & $\begin{array}{l}1.558259 \\
1.558110 \\
1.557962 \\
1.557816 \\
1.557672\end{array}$ & $\begin{array}{l}1.558219 \\
1.558070 \\
1.557922 \\
1.557776 \\
1.557632\end{array}$ & $\begin{array}{l}1.558179 \\
1.558030 \\
1.557882 \\
1.557736 \\
1.557592\end{array}$ & $\begin{array}{l}1.558139 \\
1.557990 \\
1.557842 \\
1.557696 \\
1.557552\end{array}$ & $\begin{array}{l}1.558099 \\
1.557950 \\
1.557802 \\
1.557656 \\
1.557512\end{array}$ & $\begin{array}{l}1.558060 \\
1.557911 \\
1.557762 \\
1.557616 \\
1.557472\end{array}$ & $\begin{array}{l}1.558020 \\
1.557871 \\
1.557723 \\
1.557576 \\
1.557432\end{array}$ & $\begin{array}{l}1.557980 \\
1.557831 \\
1.557683 \\
1.557537 \\
1.557392\end{array}$ & $\begin{array}{l}\text { 1. } 557940 \\
\text { 1. } 557791 \\
\text { 1. } 557643 \\
\text { 1. } 557497 \\
1.557353\end{array}$ \\
\hline & $\begin{array}{l}\text { 1. } 557689 \\
1.557547 \\
1.557407 \\
1.557269 \\
1.557132\end{array}$ & $\begin{array}{l}1.557649 \\
1.557507 \\
1.557367 \\
1.557229 \\
1.557092\end{array}$ & $\begin{array}{l}\text { 1. } 557609 \\
\text { 1. } 557467 \\
\text { A. } 557327 \\
\text { 1. } 557189 \\
\text { 1. } 557052\end{array}$ & $\begin{array}{l}1.557569 \\
1.557427 \\
1.557287 \\
1.557149 \\
1.557012\end{array}$ & $\begin{array}{l}1.557529 \\
1.557387 \\
1.557247 \\
1.557109 \\
1.556972\end{array}$ & $\begin{array}{l}\text { 1. } 557347 \\
\text { 1. } 557207 \\
\text { 1. } 557069 \\
\text { 1. } 556932\end{array}$ & $\begin{array}{l}\text { 1. } 557307 \\
\text { 1. } 557167 \\
\text { 1. } 557029 \\
\text { 1. } 556892\end{array}$ & $\begin{array}{l}1.557409 \\
1.557267 \\
1.557127 \\
1.556989 \\
1.556852\end{array}$ & & & & & $\begin{array}{l}1.557209 \\
1.557067 \\
1.556927 \\
1.556789 \\
1.556652\end{array}$ \\
\hline 6300 & $\begin{array}{l}1.556996 \\
1.556863 \\
1.556730 \\
1.556598 \\
1.556468\end{array}$ & $\begin{array}{l}1.556956 \\
1.556822 \\
1.556690 \\
1.556558 \\
1.556428\end{array}$ & $\begin{array}{l}\text { 1. } 556916 \\
\text { 1. } 556782 \\
\text { 1. } 556649 \\
1.556518 \\
1.556388\end{array}$ & $\begin{array}{l}\text { 1. } 556876 \\
1.556742 \\
1.556609 \\
1.556477 \\
1.556347\end{array}$ & $\begin{array}{l}1.556836 \\
1.556702 \\
1.556569 \\
1.556437 \\
1.556307\end{array}$ & $\begin{array}{l}1.556796 \\
1.556662 \\
1.556529 \\
1.556397 \\
1.556267\end{array}$ & $\begin{array}{l}1.556756 \\
1.556622 \\
1.556489 \\
1.556357 \\
1.556227\end{array}$ & $\begin{array}{l}1.556716 \\
1.556582 \\
1.556449 \\
1.556317 \\
1.556187\end{array}$ & $\begin{array}{l}1.556676 \\
1.556542 \\
1.556409 \\
1.556277 \\
1.556147\end{array}$ & $\begin{array}{l}\text { 1. } 556636 \\
1.556502 \\
1.556369 \\
1.556237 \\
1.556106\end{array}$ & $\begin{array}{l}1.556596 \\
1.556462 \\
1.556329 \\
1.556196 \\
1.556066\end{array}$ & & $\begin{array}{l}\text { 1. } 556516 \\
1.556381 \\
1.556248 \\
1.556116 \\
1.555986\end{array}$ \\
\hline $6480 \ldots$ & $\begin{array}{l}1.556339 \\
1.556211 \\
1.556085 \\
1.555960 \\
1.555836\end{array}$ & $\begin{array}{l}1.556299 \\
1.556171 \\
1.556045 \\
1.555919 \\
1.555795\end{array}$ & $\begin{array}{l}\text { 1. } 556259 \\
1.556131 \\
1.556005 \\
1.555879 \\
1.555755\end{array}$ & $\begin{array}{l}1.556219 \\
1.556091 \\
1.555965 \\
1.555839 \\
1.555715\end{array}$ & $\begin{array}{l}1.556178 \\
1.556050 \\
1.555924 \\
1.555799 \\
1.555675\end{array}$ & $\begin{array}{l}1.556138 \\
1.556010 \\
1.555884 \\
1.555758 \\
1.555634\end{array}$ & $\begin{array}{l}\text { 1. } 556098 \\
1.555970 \\
1.555844 \\
1.555718 \\
1.555594\end{array}$ & $\begin{array}{l}1.556058 \\
1.555930 \\
1.555804 \\
1.555678 \\
1.555554\end{array}$ & & $\begin{array}{l}\text { 1. } 555977 \\
1.555849 \\
1.555723 \\
1.555597 \\
1.555473\end{array}$ & $\begin{array}{l}1.555937 \\
1.555809 \\
1.555683 \\
1.555557 \\
1.555433\end{array}$ & $\begin{array}{l}1.555897 \\
1.555769 \\
1.555643 \\
1.555517 \\
1.555392\end{array}$ & $\begin{array}{l}\text { 1. } 555857 \\
1.555729 \\
1.555603 \\
1.555476 \\
1.555352\end{array}$ \\
\hline & $\begin{array}{l}\text { 1. } 555714 \\
1.555592 \\
1.555472 \\
1.555353 \\
1.555236\end{array}$ & $\begin{array}{l}1.555674 \\
1.555552 \\
1.555432 \\
1.555313 \\
1.555195\end{array}$ & $\begin{array}{l}1.555633 \\
1.555511 \\
1.555392 \\
1.555273 \\
1.555155\end{array}$ & $\begin{array}{l}\text { 1. } 555593 \\
1.555471 \\
1.555351 \\
1.555232 \\
1.555114\end{array}$ & $\begin{array}{l}\text { 1. } 555553 \\
1.555431 \\
1.555311 \\
1.555192 \\
1.555074\end{array}$ & $\begin{array}{l}1.555512 \\
1.555390 \\
1.555270 \\
1.555151 \\
1.555033\end{array}$ & $\begin{array}{l}\text { 1. } 555472 \\
1.555350 \\
1.555230 \\
1.555111 \\
1.554993\end{array}$ & $\begin{array}{l}1.555432 \\
1.555310 \\
1.555190 \\
1.555071 \\
1.554953\end{array}$ & & $\begin{array}{l}1.555351 \\
1.555229 \\
1.555109 \\
1.554990 \\
1.554872\end{array}$ & $\begin{array}{l}1.555311 \\
1.555189 \\
1.555068 \\
1.554949 \\
1.554831\end{array}$ & & \\
\hline $\begin{array}{l}6660 \\
6680\end{array}$ & $\begin{array}{l}1.555119 \\
1.555003 \\
1.554888 \\
1.554775 \\
1.554662\end{array}$ & $\begin{array}{l}1.555078 \\
1.554962 \\
1.554848 \\
1.554735 \\
1.554622\end{array}$ & $\begin{array}{l}1.555038 \\
1.554922 \\
1.554807 \\
1.554694 \\
1.554581\end{array}$ & $\begin{array}{l}\text { 1. } 554997 \\
1.554881 \\
1.554767 \\
1.554654 \\
1.554541\end{array}$ & $\begin{array}{l}\text { 1. } 554957 \\
1.554841 \\
1.554726 \\
1.554613 \\
1.554500\end{array}$ & $\begin{array}{l}1.554916 \\
1.554800 \\
1.554686 \\
1.554573 \\
1.554459\end{array}$ & $\begin{array}{l}\text { 1. } 554876 \\
1.554760 \\
1.554645 \\
1.554532 \\
1.554419\end{array}$ & $\begin{array}{l}1.554836 \\
1.554719 \\
1.554604 \\
1.554491 \\
1.554378\end{array}$ & $\begin{array}{l}1.554795 \\
1.554679 \\
1.554564 \\
1.554451 \\
1.554338\end{array}$ & $\begin{array}{l}1.554755 \\
1.554639 \\
1.554523 \\
1.554410 \\
1.554297\end{array}$ & $\begin{array}{l}1.554714 \\
1.554598 \\
1.554483 \\
1.554370 \\
1.554257\end{array}$ & & $\begin{array}{l}1.554633 \\
1.554517 \\
1.554402 \\
1.554289 \\
1.554176\end{array}$ \\
\hline & $\begin{array}{l}1.554552 \\
1.554442 \\
1.554332 \\
1.554224 \\
1.554117\end{array}$ & $\begin{array}{l}1.554511 \\
1.554401 \\
1.554291 \\
1.554183 \\
1.554076\end{array}$ & $\begin{array}{l}1.554470 \\
1.554360 \\
1.554251 \\
1.554143 \\
1.554036\end{array}$ & $\begin{array}{l}1.554430 \\
1.554320 \\
1.554210 \\
1.554102 \\
1.553995\end{array}$ & $\begin{array}{l}1.554389 \\
1.554279 \\
1.554169 \\
1.554061 \\
1.553954\end{array}$ & $\begin{array}{l}1.554349 \\
1.554239 \\
1.554129 \\
1.554021 \\
1.553914\end{array}$ & $\begin{array}{l}1.554308 \\
1.554198 \\
1.554088 \\
1.553980 \\
1.553873\end{array}$ & $\begin{array}{l}1.554267 \\
1.554157 \\
1.554047 \\
1.553939 \\
1.553832\end{array}$ & $\begin{array}{l}1.554227 \\
1.554117 \\
1.554007 \\
1.553899 \\
1.553792\end{array}$ & $\begin{array}{l}1.554186 \\
1.554076 \\
1.553966 \\
1.553858 \\
1.553751\end{array}$ & $\begin{array}{l}1.554416 \\
1.554035 \\
1.553925 \\
1.553817 \\
1.553710\end{array}$ & $\begin{array}{l}1.554105 \\
1.553995 \\
1.553885 \\
1.553777 \\
1.553669\end{array}$ & $\begin{array}{l}1.554064 \\
1.553954 \\
1.553844 \\
1.553736 \\
1.553629\end{array}$ \\
\hline $\begin{array}{l}6840 \\
6800 \\
6880\end{array}$ & $\begin{array}{l}1.554010 \\
1.553905 \\
1.553802 \\
1.553699 \\
1.553596\end{array}$ & $\begin{array}{l}1.553970 \\
1.553865 \\
1.553761 \\
1.553658 \\
1.553555\end{array}$ & $\begin{array}{l}\text { 1. } 553929 \\
1.553824 \\
1.553720 \\
1.553617 \\
1.553514\end{array}$ & $\begin{array}{l}\text { 1. } 553888 \\
1.553783 \\
1.553679 \\
1.553576 \\
1.553473\end{array}$ & $\begin{array}{l}1.553847 \\
1.553743 \\
1.553639 \\
1.553536 \\
1.553433\end{array}$ & $\begin{array}{l}1.553807 \\
1.553702 \\
1.553598 \\
1.553495 \\
1.553392\end{array}$ & $\begin{array}{l}\text { 1. } 553766 \\
1.553661 \\
1.553557 \\
1.553454 \\
1.553351\end{array}$ & $\begin{array}{l}1.553725 \\
1.553620 \\
1.553516 \\
1.553413 \\
1.553310\end{array}$ & $\begin{array}{l}\text { 1. } 553685 \\
1.553579 \\
1.553475 \\
1.553372 \\
1.553269\end{array}$ & $\begin{array}{l}1.553644 \\
1.553539 \\
1.553435 \\
1.553332 \\
1.553229\end{array}$ & $\begin{array}{l}1.553603 \\
1.553498 \\
1.553394 \\
1.553291 \\
1.553188\end{array}$ & $\begin{array}{l}\text { 1. } 553562 \\
1.553457 \\
1.553353 \\
1.553250 \\
1.553147\end{array}$ & $\begin{array}{l}\text { 1. } 553522 \\
\text { 1. } 553416 \\
1.553312 \\
1.553209 \\
1.553106\end{array}$ \\
\hline & $\begin{array}{l}1.553495 \\
1.553394 \\
1.553295 \\
1.553197 \\
1.553099\end{array}$ & $\begin{array}{l}1.553454 \\
1.553353 \\
1.553255 \\
1.553156 \\
1.553058\end{array}$ & $\begin{array}{l}1.553413 \\
1.553313 \\
1.553214 \\
1.553115 \\
1.553017\end{array}$ & $\begin{array}{l}\text { 1. } 553373 \\
1.553272 \\
1.553173 \\
1.553074 \\
1.552976\end{array}$ & $\begin{array}{l}\text { 1. } 553332 \\
1.553231 \\
1.553132 \\
1.553033 \\
1.552935\end{array}$ & $\begin{array}{l}1.553291 \\
1.553190 \\
1.553091 \\
1.552992 \\
1.552894\end{array}$ & $\begin{array}{l}1.553250 \\
1.553149 \\
1.553050 \\
1.552951 \\
1.552853\end{array}$ & $\begin{array}{l}1.553209 \\
1.553108 \\
1.553009 \\
1.552910 \\
1.552812\end{array}$ & $\begin{array}{l}1.553168 \\
1.553067 \\
1.552968 \\
1.552869 \\
1.552771\end{array}$ & $\begin{array}{l}1.553127 \\
1.553026 \\
1.552927 \\
1.552828 \\
1.552730\end{array}$ & $\begin{array}{l}1.553087 \\
1.552985 \\
1.552886 \\
1.525787 \\
1.552689\end{array}$ & $\begin{array}{l}1.553046 \\
1.552945 \\
1.552845 \\
1.552746 \\
1.552648\end{array}$ & $\begin{array}{l}1.553005 \\
1.552904 \\
1.552805 \\
1.552705 \\
1.552607\end{array}$ \\
\hline $\begin{array}{l}7000 \\
7020 \\
7040 \\
7060 \\
7080 \\
7100\end{array}$ & $\begin{array}{l}\text { 1. } 553002 \\
\text { 1. } 552906 \\
\text { 1. } 552811 \\
\text { 1. } 552717 \\
\text { 1. } 552623 \\
\text { 1. } 552532\end{array}$ & $\begin{array}{l}\text { 1. } 552961 \\
\text { 1. } 552865 \\
\text { 1. } 552770 \\
1.552676 \\
1.552582 \\
1.552491\end{array}$ & $\begin{array}{l}\text { 1. } 552920 \\
\text { 1. } 552824 \\
\text { 1. } 552729 \\
\text { 1. } 552635 \\
\text { 1. } 5525241 \\
\text { 1. } 552449\end{array}$ & $\begin{array}{l}\text { 1. } 552879 \\
\text { 1. } 552783 \\
\text { 1. } 552688 \\
\text { 1. } 552594 \\
\text { 1. } 5552500 \\
1.552408\end{array}$ & $\begin{array}{l}\text { 1. } 552838 \\
\text { 1. } 552742 \\
\text { 1. } 552647 \\
1.552553 \\
1.552459 \\
1.552367\end{array}$ & $\begin{array}{l}1.552797 \\
1.552701 \\
1.552606 \\
1.552512 \\
1.552418 \\
1.552326\end{array}$ & $\begin{array}{l}1.552756 \\
1.552660 \\
1.552565 \\
1.552471 \\
1.552377 \\
1.552285\end{array}$ & $\begin{array}{l}\text { 1. } 552715 \\
\text { 1. } 5521619 \\
\text { 1. } 552524 \\
1.552430 \\
1.552336 \\
1.552244\end{array}$ & $\begin{array}{l}1.552674 \\
1.552578 \\
1.552483 \\
1.552389 \\
1.55295 \\
1.552203\end{array}$ & $\begin{array}{l}\text { 1. } 552633 \\
\text { 1. } 552537 \\
\text { 1. } 552442 \\
1.552348 \\
1.552254 \\
1.552162\end{array}$ & $\begin{array}{l}1.552592 \\
1.552496 \\
1.552401 \\
1.552307 \\
1.552213 \\
1.552121\end{array}$ & $\begin{array}{l}\text { 1. } 552551 \\
\text { 1. } 5522455 \\
\text { 1. } 552360 \\
\text { 1. } 552266 \\
1.552271 \\
1.552079\end{array}$ & $\begin{array}{l}1.552510 \\
1.552414 \\
1.552319 \\
1.552225 \\
1.552130 \\
1.552038\end{array}$ \\
\hline
\end{tabular}


TABLE 2. Observed and computed refractive indrces of potassium bromide at $22^{\circ} \mathrm{C}$

\begin{tabular}{|c|c|c|c|c|c|}
\hline $\begin{array}{c}\text { Character of } \\
\text { source }\end{array}$ & $\begin{array}{c}\text { Designa- } \\
\text { tion of } \\
\text { line }\end{array}$ & $\begin{array}{l}\text { Wave- } \\
\text { lengths } \\
\text { in air }\end{array}$ & $n$ (observed) & $n$ (computed) & $\begin{array}{c}\text { Residual } \\
\text { (observed } \\
\text { minus } \\
\text { computed) }\end{array}$ \\
\hline 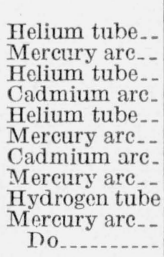 & $\begin{array}{l}\text { He red } \\
\text { Cd red } \\
\text { d } \\
\mathrm{e} \\
\mathrm{Cd} \text { green } \\
\mathrm{F} \\
\mathrm{h}_{2}\end{array}$ & $\begin{array}{c}\mu \\
0.7065188 \\
.6907496 \\
.6678149 \\
.6438470 \\
.5875618 \\
.5460740 \\
.5085824 \\
.4916036 \\
.4861327 \\
.4358342 \\
.4046563\end{array}$ & $\begin{array}{l}1.552449 \\
1.553207 \\
1.554433 \\
1.555853 \\
1.559967 \\
1.563928 \\
1.568476 \\
1.570945 \\
1.571786 \\
1.581474 \\
1.589754\end{array}$ & $\begin{array}{l}1.552447 \\
1.553212 \\
1.554430 \\
1.555853 \\
1.559966 \\
1.563930 \\
1.568479 \\
1.570935 \\
1.571789 \\
1.581475 \\
1.589753\end{array}$ & $\begin{array}{l}+2 \times 10^{-6} \\
-5 \\
+3 \\
0 \\
+1 \\
-2 \\
-3 \\
+10 \\
-3 \\
-1 \\
+1\end{array}$ \\
\hline
\end{tabular}

TABLE 3. Temperature coefficients of refractive index of potassium bromide

\begin{tabular}{|c|c|c|c}
\hline Wavelength & $-\frac{d n}{d t}$ (observed) & $-\frac{d n}{d t}$ (computed) & $\begin{array}{c}\text { Residual } \\
\text { (observed } \\
\text { minus } \\
\text { computed) }\end{array}$ \\
\hline & & & \\
\hline$\mu$ & & & \\
0.4047 & $36.75 \times 10^{-6}$ & $37.09 \times 10^{-6}$ & $-0.34 \times 10^{-6}$ \\
.4358 & 37.10 & 37.50 & -.40 \\
.4861 & 38.37 & 38.16 & +.21 \\
.4916 & 37.29 & 38.24 & -.95 \\
.5086 & 39.43 & 38.46 & +.97 \\
.5461 & 39.20 & 38.95 & +.25 \\
.5876 & 40.03 & 39.50 & +.53 \\
.6438 & 39.67 & 40.24 & -.57 \\
.6678 & 41.58 & 40.56 & +1.02 \\
.6907 & 40.41 & 40.86 & -0.45 \\
.7065 & 40.83 & 41.07 & -.24 \\
\hline
\end{tabular}

Subsequent examination of two different samples of potassium bromide by the authors verifies the possibility of even larger differences between samples. More important, differences in dispersion between samples were indicated. Figure 10 shows plots of the differences between the two other crystals studied, and the one measured upon the two spectrometers. The values designated as $P_{1}$ represent the $60^{\circ}$ sample upon which the most extensive series of measurements was made, $P_{2}$ is a $30^{\circ}$ prism, and $P_{3}$ is another $60^{\circ}$ prism. Prism $\mathrm{P}_{3}$ gave very poor imagery, and double images of the collimator slit were detected. Upon examination, this prism appeared cracked along cleaveage planes in the interior of the crystal. Single, but indistinct, images of the collimator slit appeared when the prism aperture was reduced by masking portions of the surfaces with black paper so that the radiation did not pass through the portion containing the flaws. Differences in indices amounting to as much as one unit in the third decimal place in the blue were noted for this crystal, together with a definite difference in dispersion, as indicated by the slope of the curve. The differences between $P_{2}$ and $P_{1}$ are much less pronounced, amounting to about $+6.7 \times 10^{-5}$ in the average. No difference in dispersion between these two samples was found.

\section{Conclusion}

The values of refractive indices in table 1 are thought to have errors not exceeding $\pm 5 \times 10^{-6}$. It should be understood that these index values are

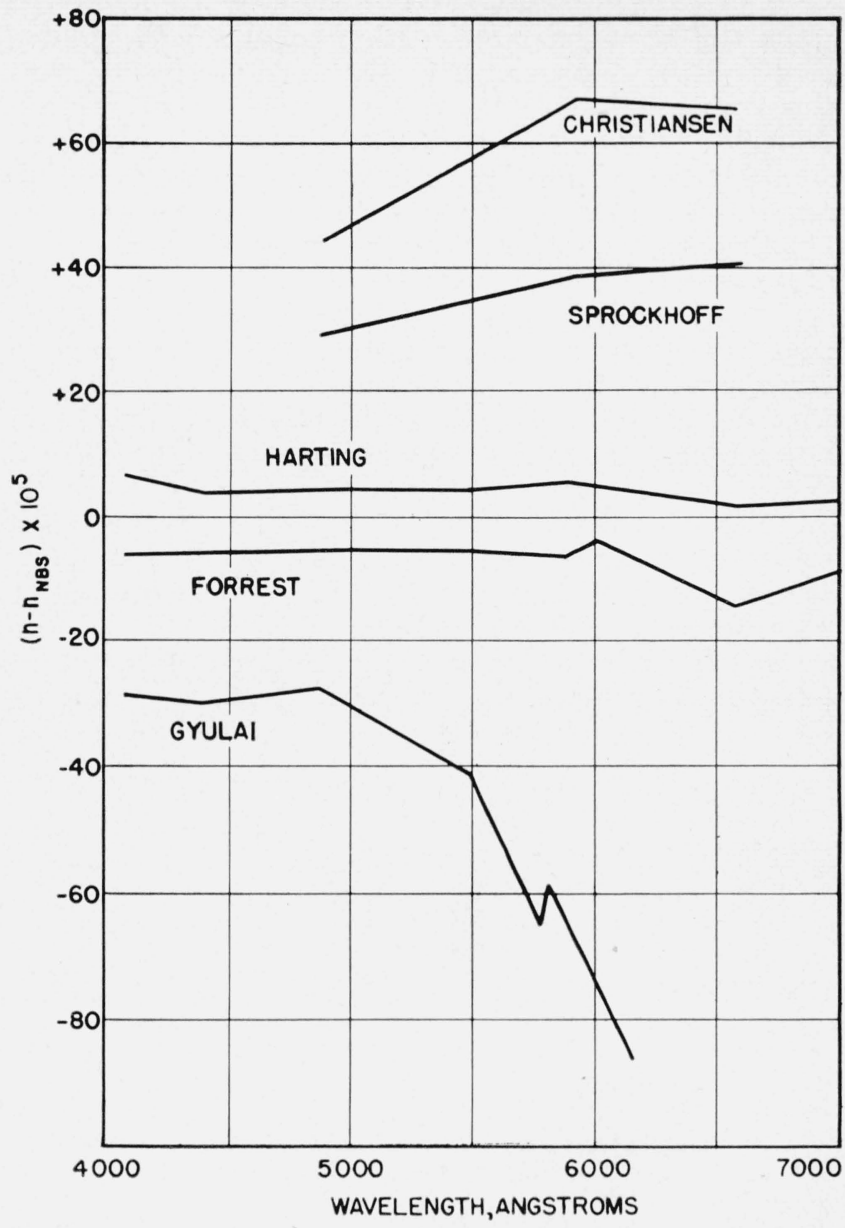

Figure 9. Comparison of indices of potassium bromide reported by different observers.

Harting's data and the NBS data are given for $20^{\circ} \mathrm{C}$. Christiansen's data at $18^{\circ} \mathrm{C}$, Sprockhoff's at $18^{\circ} \mathrm{C}$, Forrest's at $26.4^{\circ} \mathrm{C}$, and Gyulai's at $48^{\circ} \mathrm{C}$ were reduced to their $20^{\circ} \mathrm{C}$ equivalents by use of the temperature coefficients in table 3 .

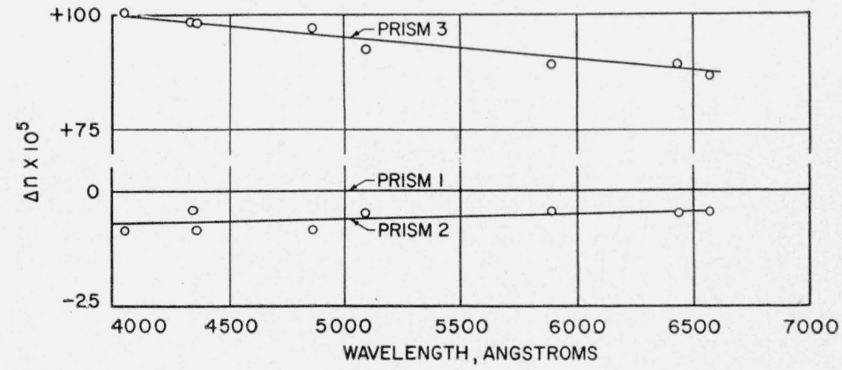

Figure 10. Comparison of different crystals of potassium bromide at $23^{\circ} \mathrm{C}$.

for the particular sample measured. The values reported by other observers are on the whole distributed rather symmetrically around the values reported in this paper. Systematic differences between different samples appear more important than hitherto realized. The reasons for these differences are not clearly understood but probably have to do with different amounts and types of impurities in the samples. The effect of impurities 
upon the ultraviolet absorption band is well known. Melvin [14], for example, mentioned in 1931 that the transmission limits of four samples of lithium fluoride varied between 1080 and $1350 \mathrm{~A}$ owing to slight traces of impurities. Shifting this absorption band, of course, influences the dispersion in this neighborhood, and it appears possible in the case of potassium bromide that this influence may extend into the visible region of the spectrum. Strain will also affect the refractive index, and it is possible that prism $P_{3}$ has some internal strain. This prism was one of the first to be made in this country, and it is possible that the same degree of care was not taken in purifying the salt and removing strain by annealing as is taken today in manufacturing such crystals. It is thought that the variation demonstrated between samples $P_{1}$ and $P_{2}$ is more nearly representative of the order of magnitude of the variation to be found between different samples of potassium bromide.

\section{References}

[1] W. S. Rodney, Dissertation, the Catholic University of America, Washington, D. C. (1951)

[2] H. Harting, Z. Instrumentenk. 63, 125-136 (1943).

[3] J. W. Forrest, J. Opt. Soc. Am. 32, 382 (1942).

[4] G. N. Ramachandran, Proc. Indian Acad. Sci. [A] 25, 481 (1947).

[5] T. Radhakrishnan, Proc. Indian Acad. Sci. [A] 31, 224 (1950).

[6] J. Guild and G. W. Watts, Proc. Opt. Conv. II, 937 (1926).

[7] J. Guild, Proc. Phys. Soc. (London) 29, 311 (1916).

[8] R. Glazebrook, Dictionary of Applied Physies IV.

[9] L. W. Tilton, BS J. Research 2, 909 (1929) RP64.

[10] L. W. Tilton, J. Research NBS 14, 393 (1935) RP393.

[11] H. Topsoe and C. Christiansen, Ann. chim. et phys. 1, 5-99 (1874)

[12] M. Sprockhoff, Neues Jahr. Mineral. Geol., Beilage Rd. 18, 117-154'(1904).

[13] Z. Gyulai, Z. Physik 46, 80 (1927)

[14] E. Melvin, Phys. Rev. 37, 1230 (1931).

Washington, D. C., July 30, 1952. 